\title{
Reactive Metabolites from Thiazole-containing Drugs: Quantum Chemical Insights into Biotransformation and Toxicity
}

\author{
Chaitanya K. Jaladanki, ${ }^{1}$ Samima Khatun, ${ }^{1}$ Holger Gohlke, ${ }^{2,3}$ Prasad V. Bharatam ${ }^{1 *}$ \\ ${ }^{1}$ Department of Medicinal Chemistry, National Institute of Pharmaceutical Education and Research (NIPER), \\ Sector - 67, S. A. S. Nagar (Mohali), 160062 Punjab, India. \\ ${ }^{2}$ Heinrich-Heine-Universität Düsseldorf, Institut für Pharmazeutische und Medizinische Chemie, \\ Universitätsstr. 1, 40225 Düsseldorf, Germany \\ ${ }^{3}$ Forschungszentrum Jülich GmbH, John von Neumann Institute for Computing (NIC),Jülich Supercomputing \\ Centre (JSC), and Institute of Biological Information Processing (IBI-7: Structural Biochemistry), Wilhelm- \\ Johnen-Straße, 52425 Jülich, Germany \\ *Corresponding Author \\ Prof. Prasad V. Bharatam, \\ Department of Medicinal Chemistry \\ E-mail: pvbharatam@niper.ac.in
}

\section{Supporting Information}

Table S1. A list of drugs or lead molecules containing thiazole scaffold.

Table S2. List of nucleophilic residues present in the vicinity of active site in CYP 2C9, 2D6, 3A4, 2C19 and 2E1 (up to $6 \AA$ ).

Table S3. The relative free energies $(\Delta G)$ and the global electrophilicity indices $(\omega)$ of the reactive metabolites. The activation energies $\left(E_{\mathrm{a}}\right)$ for the reaction of the $\mathbf{R M}$ with $\mathrm{MeO}^{-}$.

Table S4.The absolute energies (Gibbs free energy) in Hartree of the various species employed in the manuscript. The values were obtained using B3LYP(SCRF)/6-311++G(d,p)// B3LYP/6-31+G(d) basis set $(\varepsilon=5.9)$.

Figure S1. 2D structures of Thiazole compounds (as listed in Table S1).

Figure S2. Optimized geometries of transition states (TS-E, TS-S, TS-N, TS-A) and product complexes (PCE, PC-S, PC-N, PC-A) along the various reaction pathways of thiazole. The values are on the doublet (quartet) potential energy surface. All bond lengths are in Ångstrom $(\AA)$ and bond angles are in degrees $\left(^{\circ}\right)$.

Figure S3. Energy profile comparison between TZ (right side) and ATZ (left side) (epoxidation, $S$-oxidation, $\mathrm{N}$-oxidation, oxaziridine and $\mathrm{N}$-hydroxylation formation in the presence of $\mathrm{Cpd}$ I) on the doublet potential energy surface of $\mathrm{Cpd}$ I. The values are obtained using B3LYP(SCRF)/6$311++\mathrm{G}(\mathrm{d}, \mathrm{p}) / / \mathrm{B} 3 \mathrm{LYP} / 6-31+\mathrm{G}(\mathrm{d})$.

Figure S4. Potential energy profile of AMTZ (hydroxylation, epoxidation, $S$-oxidation, and $N$-oxidation) on the doublet state of Cpd I. All the values are obtained using B3LYP(SCRF)/6$311++G(d, p) / / B 3 L Y P / 6-31+G(d)$ level of quantum chemistry.

Figure S5. A comparative potential energy profile of TZ (epoxidation, $S$-oxidation, and $N$-oxidation) on the doublet (right) quartet state (left) of Cpd I. All the values are obtained using B3LYP(SCRF)/6- 
$311++\mathrm{G}(\mathrm{d}, \mathrm{p}) / / \mathrm{B} 3 \mathrm{LYP} / 6-31+\mathrm{G}(\mathrm{d})$ level of quantum chemistry. Since the values along the quartet PE surface are consistently higher than that of the double PE surface, the discussion in the main text is limited to the results according to doublet PE surface.

Figure S6. The docking poses of sudoxicam (A) favoring epoxidation, $\mathrm{C}^{5}$ atom of the thiazole ring is pointed toward the $\mathrm{Fe}=\mathrm{O}$ center and meloxicam $(\mathbf{B})$ favoring hydroxylation, the methyl group at $\mathrm{C}^{5}$ of thiazole ring is pointed towards the $\mathrm{Fe}=\mathrm{O}$ center in active site of CYP3A4.

Figure S7. Potential energy profile diagram of C4-AMTZ (hydroxylation, epoxidation and $S$-oxidation) on the doublet state of Cpd I. All the values are obtained using B3LYP(SCRF)/6$311++\mathrm{G}(\mathrm{d}, \mathrm{p}) / / \mathrm{B} 3 \mathrm{LYP} / 6-31+\mathrm{G}(\mathrm{d})$ level of quantum chemistry.

Figure S8. The transition states involved in the rearrangement reaction from M1 to I10 and nucleophilic reaction between $\mathrm{M1}$ and $\mathrm{MeO}^{-}$(TS2) and $\mathrm{MeoS}^{-}$(TS3). All of the distances are in angstroms $(\AA)$ and angles are in degree $\left(^{\circ}\right)$.

Figure S9. Participation of orbitals in the electron transfer during epoxidation and $S$-oxidation pathways. (a) Cartoon representation of orbital overlap of the $\pi$-bond with the $\pi *_{x z}$ orbital of heme in the doublet state (left) and with $\sigma *_{\mathrm{z}^{2}}$ of heme in the quartet state (right). (b) Cartoon representation of the orbital overlap of $S$-lone pair with $\pi *_{\mathrm{xz}}$ of heme in the doublet state (left) and with $\sigma *_{z^{2}}$ of heme in the quartet state (right). 
Table S1. A list of drugs or lead molecules containing thiazole scaffold . @

\begin{tabular}{|c|c|c|c|c|}
\hline S.No. & Drug & Description & Docked & SMILES Notation of the drugs \\
\hline 1. & $\begin{array}{l}\text { 2,2'-Dibenzothiazyl } \\
\text { disulfide }\end{array}$ & Allergen and dermatological sensitizer & Yes & $\mathrm{S}(\mathrm{SC} 1=\mathrm{NC} 2=\mathrm{CC}=\mathrm{CC}=\mathrm{C} 2 \mathrm{~S} 1) \mathrm{C} 1=\mathrm{NC} 2=\mathrm{CC}=\mathrm{CC}=\mathrm{C} 2 \mathrm{~S} 1$ \\
\hline 2. & 2-mercaptobenzothiazole & Antiinfective agent & Yes & $\mathrm{S}=\mathrm{C} 1 \mathrm{NC} 2=\mathrm{C}(\mathrm{S} 1) \mathrm{C}=\mathrm{CC}=\mathrm{C} 2$ \\
\hline 3. & 4SC-203 & Multi-kinase inhibitor with potential antineoplastic activity & No & $\begin{array}{l}\mathrm{COC} 1=\mathrm{CC}=\mathrm{C}(\mathrm{C}) \mathrm{C}=\mathrm{C} 1 \mathrm{NC}(=\mathrm{O}) \mathrm{NC} 1=\mathrm{NC} 2=\mathrm{CC}=\mathrm{C}(\mathrm{NC} 3=\mathrm{NC}=\mathrm{NC} 4=\mathrm{CC}(\mathrm{OCCCN} 5 \mathrm{CC} \\
\mathrm{N}(\mathrm{C}) \mathrm{CC} 5)=\mathrm{C}(\mathrm{OC}) \mathrm{C}=\mathrm{C} 34) \mathrm{C}=\mathrm{C} 2 \mathrm{~S} 1\end{array}$ \\
\hline 4. & AT-001 & $\begin{array}{l}\text { Under investigation in clinical trial for long-term } \\
\text { preservation of brain health in aging }\end{array}$ & Yes & $\mathrm{OC}(=\mathrm{O}) \mathrm{CC} 1=\mathrm{NN}(\mathrm{CC} 2=\mathrm{NC} 3=\mathrm{C}(\mathrm{S} 2) \mathrm{C}=\mathrm{CC}(=\mathrm{C} 3) \mathrm{C}(\mathrm{F})(\mathrm{F}) \mathrm{F}) \mathrm{C}(=\mathrm{O}) \mathrm{C} 2=\mathrm{C} 1 \mathrm{~N}=\mathrm{CC}=\mathrm{N} 2$ \\
\hline 5. & Abafungin & $\begin{array}{l}\text { Investigated for use/treatment in fungal infections, bacterial } \\
\text { infection, skin infections/disorders, and onychomycosis }\end{array}$ & Yes & $\mathrm{CC} 1=\mathrm{CC}=\mathrm{C}(\mathrm{OC} 2=\mathrm{CC}=\mathrm{CC}=\mathrm{C} 2 \mathrm{C} 2=\mathrm{CSC}(\mathrm{N}=\mathrm{C} 3 \mathrm{NCCCN} 3)=\mathrm{N} 2) \mathrm{C}(\mathrm{C})=\mathrm{C} 1$ \\
\hline 6. & Acotiamide & Used in the treatment of functional dyspepsia & Yes & $\mathrm{COC} 1=\mathrm{C}(\mathrm{OC}) \mathrm{C}=\mathrm{C}(\mathrm{C}(=\mathrm{O}) \mathrm{NC} 2=\mathrm{NC}(=\mathrm{CS} 2) \mathrm{C}(=\mathrm{O}) \mathrm{NCCN}(\mathrm{C}(\mathrm{C}) \mathrm{C}) \mathrm{C}(\mathrm{C}) \mathrm{C}) \mathrm{C}(\mathrm{O})=\mathrm{C} 1$ \\
\hline 7. & Alagebrium & $\begin{array}{l}\text { Investigated for the treatment and prevention of aging, heart } \\
\text { failure, physical activity, diabetic nephropathy, and } \\
\text { cardiovascular diseases }\end{array}$ & Yes & $\mathrm{CC} 1=\mathrm{C}(\mathrm{C})[\mathrm{N}+](\mathrm{CC}(=\mathrm{O}) \mathrm{C} 2=\mathrm{CC}=\mathrm{CC}=\mathrm{C} 2)=\mathrm{CS} 1$ \\
\hline 8. & Alpelisib & PI3K inhibitor with potent antitumor activity & Yes & $\begin{array}{l}\mathrm{CC} 1=\mathrm{C}(\mathrm{SC}(\mathrm{NC}(=\mathrm{O}) \mathrm{N} 2 \mathrm{CCC}[\mathrm{C} @ \mathrm{H}] 2 \mathrm{C}(\mathrm{N})=\mathrm{O})=\mathrm{N} 1) \mathrm{C} 1=\mathrm{CC}(=\mathrm{NC}=\mathrm{C} 1) \mathrm{C}(\mathrm{C})(\mathrm{C}) \mathrm{C}(\mathrm{F})(\mathrm{F}) \\
\mathrm{F}\end{array}$ \\
\hline 9. & Amcasertib & $\begin{array}{l}\text { Cancer stemness kinase inhibitor with potential anticancer } \\
\text { activity }\end{array}$ & No & $\begin{array}{l}\mathrm{CCN}(\mathrm{CC}) \mathrm{CCNC}(=\mathrm{O}) \mathrm{C} 1=\mathrm{C}(\mathrm{C}) \mathrm{NC}(\mathrm{C}=\mathrm{C} 2 / \mathrm{C}(=\mathrm{O}) \mathrm{NC} 3=\mathrm{CC}=\mathrm{C}(\mathrm{C}=\mathrm{C} 23) \mathrm{C} 2=\mathrm{CSC}(=\mathrm{N} 2) \mathrm{C} \\
2=\mathrm{CC}=\mathrm{CC}=\mathrm{C} 2)=\mathrm{C} 1 \mathrm{C}\end{array}$ \\
\hline 10. & Arotinolol & $\begin{array}{l}\text { Investigated for its potential to be used as an anti- } \\
\text { hypertensive agent }\end{array}$ & Yes & $\mathrm{CC}(\mathrm{C})(\mathrm{C}) \mathrm{NCC}(\mathrm{O}) \mathrm{CSC} 1=\mathrm{NC}(=\mathrm{CS} 1) \mathrm{C} 1=\mathrm{CC}=\mathrm{C}(\mathrm{S} 1) \mathrm{C}(\mathrm{N})=\mathrm{O}$ \\
\hline 11. & Avatrombopag & $\begin{array}{l}\text { Platelet Thrombopoietin Receptor (TPOR; MPL) agonist, } \\
\text { with potential megakaryopoiesis stimulating activity }\end{array}$ & No & $\begin{array}{l}\mathrm{OC}(=\mathrm{O}) \mathrm{C} 1 \mathrm{CCN}(\mathrm{CC} 1) \mathrm{C} 1=\mathrm{C}(\mathrm{Cl}) \mathrm{C}=\mathrm{C}(\mathrm{C}=\mathrm{N} 1) \mathrm{C}(=\mathrm{O}) \mathrm{NC} 1=\mathrm{NC}(\mathrm{C} 2=\mathrm{CC}(\mathrm{Cl})=\mathrm{CS} 2)=\mathrm{C}(\mathrm{S} 1 \\
) \mathrm{N} 1 \mathrm{CCN}(\mathrm{CC} 1) \mathrm{C} 1 \mathrm{CCCCC} 1\end{array}$ \\
\hline 12. & Aztreonam & $\begin{array}{l}\text { Used primarily to treat infections caused by Gram-negative } \\
\text { bacteria }\end{array}$ & Yes & $\begin{array}{l}\mathrm{C}[\mathrm{C} @ \mathrm{H}] 1[\mathrm{C} @ \mathrm{H}](\mathrm{NC}(=\mathrm{O}) \mathrm{C}(=\mathrm{N} / \mathrm{OC}(\mathrm{C})(\mathrm{C}) \mathrm{C}(=\mathrm{O}) \mathrm{O}) \mathrm{C} 2=\mathrm{CSC}([\mathrm{NH} 3+])=\mathrm{N} 2) \mathrm{C}(=\mathrm{O}) \mathrm{N} 1 \\
\mathrm{~S}([\mathrm{O}-])(=\mathrm{O})=\mathrm{O}\end{array}$ \\
\hline 13. & BMS-986141 & Anti-thrombotic activity & No & $\begin{array}{l}\mathrm{COC} 1=\mathrm{NN} 2 \mathrm{C}=\mathrm{C}(\mathrm{N}=\mathrm{C} 2 \mathrm{~S} 1) \mathrm{C} 1=\mathrm{CC} 2=\mathrm{C}(\mathrm{O} 1) \mathrm{C}=\mathrm{C}(\mathrm{OC}) \mathrm{C}=\mathrm{C} 2 \mathrm{OCC} 1=\mathrm{CSC}(=\mathrm{N} 1) \mathrm{C} 1=\mathrm{CC} \\
=\mathrm{C}(\mathrm{C}=\mathrm{C} 1) \mathrm{C}(=\mathrm{O}) \mathrm{N}(\mathrm{C}) \mathrm{C}\end{array}$ \\
\hline 14. & Bleomycin & Antineoplastic, especially for solid tumors & No & $\begin{array}{l}\mathrm{C}[\mathrm{C} @ @ \mathrm{H}](\mathrm{O})[\mathrm{C} @ \mathrm{H}](\mathrm{NC}(=\mathrm{O})[\mathrm{C} @ @ \mathrm{H}](\mathrm{C})[\mathrm{C} @ \mathrm{H}](\mathrm{O})[\mathrm{C} @ @ \mathrm{H}](\mathrm{C}) \mathrm{NC}(=\mathrm{O})[\mathrm{C} @ @ \mathrm{H}]( \\
\mathrm{NC}(=\mathrm{O}) \mathrm{C} 1=\mathrm{C}(\mathrm{C}) \mathrm{C}(\mathrm{N})=\mathrm{NC}(=\mathrm{N} 1)[\mathrm{C} @ \mathrm{H}](\mathrm{CC}(\mathrm{N})=\mathrm{O}) \mathrm{NC}[\mathrm{C} @ \mathrm{H}](\mathrm{N}) \mathrm{C}(\mathrm{N})=\mathrm{O})[\mathrm{C} @ @ \mathrm{H}]( \\
\mathrm{OC} 1 \mathrm{OC}(\mathrm{CO}) \mathrm{C}(\mathrm{O}) \mathrm{C}(\mathrm{O}) \mathrm{C} 1 \mathrm{OC} 1 \mathrm{OC}(\mathrm{CO}) \mathrm{C}(\mathrm{O}) \mathrm{C}(\mathrm{OC}(\mathrm{N})=\mathrm{O}) \mathrm{C} 1 \mathrm{O}) \mathrm{C} 1=\mathrm{CN}=\mathrm{CN} 1) \mathrm{C}(=\mathrm{O}) \mathrm{N} \\
\mathrm{CCC} 1=\mathrm{NC}(=\mathrm{CS} 1) \mathrm{C} 1=\mathrm{NC}(=\mathrm{CS} 1) \mathrm{C}(=\mathrm{O}) \mathrm{NCCC}[\mathrm{S}+](\mathrm{C}) \mathrm{C}\end{array}$ \\
\hline 15. & Brecanavir & Used for the treatment of HIV & No & $\begin{array}{l}{[\mathrm{H}][\mathrm{C} @] 12 \mathrm{OCC}[\mathrm{C} @ @] 1([\mathrm{H}])[\mathrm{C} @ \mathrm{H}](\mathrm{CO} 2) \mathrm{OC}(=\mathrm{O}) \mathrm{N}[\mathrm{C} @ @ \mathrm{H}](\mathrm{CC} 1=\mathrm{CC}=\mathrm{C}(\mathrm{OCC} 2=} \\
\mathrm{CSC}(\mathrm{C})=\mathrm{N} 2) \mathrm{C}=\mathrm{C} 1)[\mathrm{C} @ \mathrm{H}](\mathrm{O}) \mathrm{CN}(\mathrm{CC}(\mathrm{C}) \mathrm{C}) \mathrm{S}(=\mathrm{O})(=\mathrm{O}) \mathrm{C} 1=\mathrm{CC} 2=\mathrm{C}(\mathrm{OCO} 2) \mathrm{C}=\mathrm{C} 1\end{array}$ \\
\hline 16. & Cambendazole & Anthelmintic activity & Yes & $\mathrm{CC}(\mathrm{C}) \mathrm{OC}(=\mathrm{O}) \mathrm{NC} 1=\mathrm{CC} 2=\mathrm{C}(\mathrm{NC}(=\mathrm{N} 2) \mathrm{C} 2=\mathrm{CSC}=\mathrm{N} 2) \mathrm{C}=\mathrm{C} 1$ \\
\hline 17. & Cardarine & $\begin{array}{l}\text { Peroxisome proliferator-activator receptor-delta agonist for } \\
\text { the potential treatment of dyslipidemia }\end{array}$ & Yes & $\mathrm{CC} 1=\mathrm{C}(\mathrm{CSC} 2=\mathrm{CC}(\mathrm{C})=\mathrm{C}(\mathrm{OCC}(\mathrm{O})=\mathrm{O}) \mathrm{C}=\mathrm{C} 2) \mathrm{SC}(=\mathrm{N} 1) \mathrm{C} 1=\mathrm{CC}=\mathrm{C}(\mathrm{C}=\mathrm{C} 1) \mathrm{C}(\mathrm{F})(\mathrm{F}) \mathrm{F}$ \\
\hline 18. & Cefdinir & $\begin{array}{l}\text { Proven to be effective for the treatment of common bacterial } \\
\text { infections in the ear, sinus, throat, lungs, and skin }\end{array}$ & Yes & $\begin{array}{l}{[\mathrm{H}][\mathrm{C} @] 12 \mathrm{SCC}(\mathrm{C}=\mathrm{C})=\mathrm{C}(\mathrm{N} 1 \mathrm{C}(=\mathrm{O})[\mathrm{C} @ \mathrm{H}] 2 \mathrm{NC}(=\mathrm{O}) \mathrm{C}(=\mathrm{N} / \mathrm{O}) \mathrm{C} 1=\mathrm{CSC}(\mathrm{N})=\mathrm{N} 1) \mathrm{C}(\mathrm{O})=} \\
\mathrm{O}\end{array}$ \\
\hline
\end{tabular}




\begin{tabular}{|c|c|c|c|c|}
\hline 19. & Cefditoren & Broad spectrum antibiotic & Yes & $\begin{array}{l}{[\mathrm{H}][\mathrm{C} @] 12 \mathrm{SCC}(\mathrm{C}=\mathrm{C} / \mathrm{C} 3=\mathrm{C}(\mathrm{C}) \mathrm{N}=\mathrm{CS} 3)=\mathrm{C}(\mathrm{N} 1 \mathrm{C}(=\mathrm{O})[\mathrm{C} @ \mathrm{H}] 2 \mathrm{NC}(=\mathrm{O}) \mathrm{C}(=\mathrm{N} / \mathrm{OC}) \mathrm{C} 1=\mathrm{C}} \\
\mathrm{SC}(\mathrm{N})=\mathrm{N} 1) \mathrm{C}(\mathrm{O})=\mathrm{O}\end{array}$ \\
\hline 20. & Cefepime & Severe nosocomial pneumonia & Yes & $\begin{array}{l}\mathrm{CON}=\mathrm{C}(/ \mathrm{C}(=\mathrm{O}) \mathrm{N}[\mathrm{C} @ @ \mathrm{H}] 1 \mathrm{C}(=\mathrm{O}) \mathrm{N} 2[\mathrm{C} @] 1([\mathrm{H}]) \mathrm{SCC}(\mathrm{C}[\mathrm{N}+] 1(\mathrm{C}) \mathrm{CCCC} 1)=\mathrm{C} 2 \mathrm{C}([\mathrm{O}- \\
])=\mathrm{O}) \mathrm{C} 1=\mathrm{CSC}(\mathrm{N})=\mathrm{N} 1\end{array}$ \\
\hline 21. & Cefiderocol & $\begin{array}{l}\text { Indicated for the treatment of complicated urinary tract } \\
\text { infections with or without pyelonephritis }\end{array}$ & No & $\begin{array}{l}{[\mathrm{H}][\mathrm{C} @] 12 \mathrm{SCC}(\mathrm{C}[\mathrm{N}+] 3(\mathrm{CCNC}(=\mathrm{O}) \mathrm{C} 4=\mathrm{C}(\mathrm{Cl}) \mathrm{C}(\mathrm{O})=\mathrm{C}(\mathrm{O}) \mathrm{C}=\mathrm{C} 4) \mathrm{CCCC} 3)=\mathrm{C}(\mathrm{N} 1 \mathrm{C}(=\mathrm{O}} \\
)[\mathrm{C} @ \mathrm{H}] 2 \mathrm{NC}(=\mathrm{O}) \mathrm{C}(=\mathrm{N} / \mathrm{OC}(\mathrm{C})(\mathrm{C}) \mathrm{C}(\mathrm{O})=\mathrm{O}) \mathrm{C} 1=\mathrm{CSC}(\mathrm{N})=\mathrm{N} 1) \mathrm{C}([\mathrm{O}-])=\mathrm{O}\end{array}$ \\
\hline 22. & Cefixime & Used in the treatment of bacterial infection & Yes & $\begin{array}{l}{[\mathrm{H}][\mathrm{C} @] 12 \mathrm{SCC}(\mathrm{C}=\mathrm{C})=\mathrm{C}(\mathrm{N} 1 \mathrm{C}(=\mathrm{O})[\mathrm{C} @ \mathrm{H}] 2 \mathrm{NC}(=\mathrm{O}) \mathrm{C}(=\mathrm{N} / \mathrm{OCC}(\mathrm{O})=\mathrm{O}) \mathrm{C} 1=\mathrm{CSC}(\mathrm{N})=} \\
\mathrm{N} 1) \mathrm{C}(\mathrm{O})=\mathrm{O}\end{array}$ \\
\hline 23. & Cefmenoxime & Beta-lactam cephalosporin antibiotic & Yes & $\begin{array}{l}{[\mathrm{H}][\mathrm{C} @] 12 \mathrm{SCC}(\mathrm{CSC} 3=\mathrm{NN}=\mathrm{NN} 3 \mathrm{C})=\mathrm{C}(\mathrm{N} 1 \mathrm{C}(=\mathrm{O})[\mathrm{C} @ \mathrm{H}] 2 \mathrm{NC}(=\mathrm{O}) \mathrm{C}(=\mathrm{N} / \mathrm{OC}) \mathrm{C} 1=\mathrm{CSC}} \\
(\mathrm{N})=\mathrm{N} 1) \mathrm{C}(\mathrm{O})=\mathrm{O}\end{array}$ \\
\hline 24. & Cefotaxime & $\begin{array}{l}\text { Broad spectrum of activity against Gram-positive and Gram- } \\
\text { negative aerobic and anaerobic bacteria }\end{array}$ & Yes & $\begin{array}{l}{[\mathrm{H}][\mathrm{C} @] 12 \mathrm{SCC}(\mathrm{COC}(\mathrm{C})=\mathrm{O})=\mathrm{C}(\mathrm{N} 1 \mathrm{C}(=\mathrm{O})[\mathrm{C} @ \mathrm{H}] 2 \mathrm{NC}(=\mathrm{O}) \mathrm{C}(=\mathrm{N} / \mathrm{OC}) \mathrm{C} 1=\mathrm{CSC}(\mathrm{N})=\mathrm{N}} \\
1) \mathrm{C}(\mathrm{O})=\mathrm{O}\end{array}$ \\
\hline 25. & Cefotiam & $\begin{array}{l}\text { Broad spectrum activity against Gram-positive and Gram- } \\
\text { negative bacteria }\end{array}$ & No & $\begin{array}{l}{[\mathrm{H}][\mathrm{C} @] 12 \mathrm{SCC}(\mathrm{CSC} 3=\mathrm{NN}=\mathrm{NN} 3 \mathrm{CCN}(\mathrm{C}) \mathrm{C})=\mathrm{C}(\mathrm{N} 1 \mathrm{C}(=\mathrm{O})[\mathrm{C} @ \mathrm{H}] 2 \mathrm{NC}(=\mathrm{O}) \mathrm{CC} 1=\mathrm{CSC}(} \\
\mathrm{N})=\mathrm{N} 1) \mathrm{C}(\mathrm{O})=\mathrm{O}\end{array}$ \\
\hline 26. & Cefpirome & Active against Enterobacteriacae & Yes & $\begin{array}{l}{[\mathrm{H}][\mathrm{C} @] 12 \mathrm{SCC}(\mathrm{C}[\mathrm{N}+] 3=\mathrm{C} 4 \mathrm{CCCC} 4=\mathrm{CC}=\mathrm{C} 3)=\mathrm{C}(\mathrm{N} 1 \mathrm{C}(=\mathrm{O})[\mathrm{C} @ \mathrm{H}] 2 \mathrm{NC}(=\mathrm{O}) \mathrm{C}(=\mathrm{N} / \mathrm{OC}} \\
) \mathrm{C} 1=\mathrm{CSC}(\mathrm{N})=\mathrm{N} 1) \mathrm{C}([\mathrm{O}-])=\mathrm{O}\end{array}$ \\
\hline 27. & Cefpodoxime & $\begin{array}{l}\text { Effective against most Gram positive and Gram negative } \\
\text { bacteria. }\end{array}$ & Yes & $\begin{array}{l}{[\mathrm{H}][\mathrm{C} @] 12 \mathrm{SCC}(\mathrm{COC})=\mathrm{C}(\mathrm{N} 1 \mathrm{C}(=\mathrm{O})[\mathrm{C} @ \mathrm{H}] 2 \mathrm{NC}(=\mathrm{O}) \mathrm{C}(=\mathrm{N} / \mathrm{OC}) \mathrm{C} 1=\mathrm{CSC}(\mathrm{N})=\mathrm{N} 1) \mathrm{C}(\mathrm{O})} \\
=\mathrm{O}\end{array}$ \\
\hline 28. & Ceftarolinefosamil & Broad spectrum activity against Gram-positive pathogens & No & $\begin{array}{l}{[\mathrm{H}][\mathrm{C} @] 12 \mathrm{SCC}(\mathrm{SC} 3=\mathrm{NC}(=\mathrm{CS} 3) \mathrm{C} 3=\mathrm{CC}=[\mathrm{N}+](\mathrm{C}) \mathrm{C}=\mathrm{C} 3)=\mathrm{C}(\mathrm{N} 1 \mathrm{C}(=\mathrm{O})[\mathrm{C} @ \mathrm{H}] 2 \mathrm{NC}(=} \\
\mathrm{O}) \mathrm{C}(=\mathrm{N} / \mathrm{OCC}) \mathrm{C} 1=\mathrm{NSC}(\mathrm{NP}(\mathrm{O})(\mathrm{O})=\mathrm{O})=\mathrm{N} 1) \mathrm{C}([\mathrm{O}-])=\mathrm{O}\end{array}$ \\
\hline 29. & Ceftazidime & $\begin{array}{l}\text { Activity against many Gram-negative bacteria resistant to } \\
\text { other drugs }\end{array}$ & No & $\begin{array}{l}{[\mathrm{O}-} \\
] \mathrm{C}(=\mathrm{O}) \mathrm{C} 1=\mathrm{C}(\mathrm{CS}[\mathrm{C} @] 2([\mathrm{H}])[\mathrm{C} @ \mathrm{H}](\mathrm{NC}(=\mathrm{O}) \mathrm{C}(=\mathrm{N} / \mathrm{OC}(\mathrm{C})(\mathrm{C}) \mathrm{C}(\mathrm{O})=\mathrm{O}) \mathrm{C} 3=\mathrm{CSC}(\mathrm{N})=\mathrm{N} \\
3) \mathrm{C}(=\mathrm{O}) \mathrm{N} 12) \mathrm{C}[\mathrm{N}+] 1=\mathrm{CC}=\mathrm{CC}=\mathrm{C} 1\end{array}$ \\
\hline 30. & Ceftibuten & $\begin{array}{l}\text { Used to treat acute bacterial exacerbations of chronic } \\
\text { bronchitis (ABECB), acute bacterial otitis media, pharyngitis, } \\
\text { and tonsilitis. }\end{array}$ & Yes & $\begin{array}{l}{[\mathrm{H}][\mathrm{C} @] 12 \mathrm{SCC}=\mathrm{C}(\mathrm{N} 1 \mathrm{C}(=\mathrm{O})[\mathrm{C} @ \mathrm{H}] 2 \mathrm{NC}(=\mathrm{O}) \mathrm{C}(=\mathrm{C} / \mathrm{CC}(\mathrm{O})=\mathrm{O}) \mathrm{C} 1=\mathrm{CSC}(\mathrm{N})=\mathrm{N} 1) \mathrm{C}(\mathrm{O})} \\
=\mathrm{O}\end{array}$ \\
\hline 31. & Ceftiofur & Bactericidal activity against Gram positive aerobes & Yes & $\begin{array}{l}{[\mathrm{H}][\mathrm{C} @] 12 \mathrm{SCC}(\mathrm{CSC}(=\mathrm{O}) \mathrm{C} 3=\mathrm{CC}=\mathrm{CO} 3)=\mathrm{C}(\mathrm{N} 1 \mathrm{C}(=\mathrm{O})[\mathrm{C} @ \mathrm{H}] 2 \mathrm{NC}(=\mathrm{O}) \mathrm{C}(=\mathrm{N} / \mathrm{OC}) \mathrm{C} 1=} \\
\mathrm{CSC}(\mathrm{N})=\mathrm{N} 1) \mathrm{C}(\mathrm{O})=\mathrm{O}\end{array}$ \\
\hline 32. & Ceftizoxime & $\begin{array}{l}\text { Active against a wide range of both aerobic and anaerobic } \\
\text { Gram-positive and Gram-negative organisms }\end{array}$ & Yes & {$[\mathrm{H}][\mathrm{C} @] 12 \mathrm{SCC}=\mathrm{C}(\mathrm{N} 1 \mathrm{C}(=\mathrm{O})[\mathrm{C} @ \mathrm{H}] 2 \mathrm{NC}(=\mathrm{O}) \mathrm{C}(=\mathrm{N} / \mathrm{OC}) \mathrm{C} 1=\mathrm{CSC}(\mathrm{N})=\mathrm{N} 1) \mathrm{C}(\mathrm{O})=\mathrm{O}$} \\
\hline 33. & Ceftriaxone & Bactericidal activity & Yes & $\begin{array}{l}{[\mathrm{H}][\mathrm{C} @] 12 \mathrm{SCC}(\mathrm{CSC} 3=\mathrm{NC}(=\mathrm{O}) \mathrm{C}(=\mathrm{O}) \mathrm{NN} 3 \mathrm{C})=\mathrm{C}(\mathrm{N} 1 \mathrm{C}(=\mathrm{O})[\mathrm{C} @ \mathrm{H}] 2 \mathrm{NC}(=\mathrm{O}) \mathrm{C}(=\mathrm{N} / \mathrm{OC}} \\
) \mathrm{C} 1=\mathrm{CSC}(\mathrm{N})=\mathrm{N} 1) \mathrm{C}(\mathrm{O})=\mathrm{O}\end{array}$ \\
\hline 34. & Ciluprevir & $\begin{array}{l}\text { Investigated for use/treatment in Hepatitis C Virus (HCV) } \\
\text { infection }\end{array}$ & No & $\begin{array}{l}\mathrm{COC} 1=\mathrm{CC} 2=\mathrm{C}(\mathrm{C}=\mathrm{C} 1) \mathrm{C}(\mathrm{O}[\mathrm{C} @ \mathrm{H}] 1 \mathrm{CN} 3 \mathrm{C}(=\mathrm{O})[\mathrm{C} @ \mathrm{H}](\mathrm{CCCCCC}=\mathrm{C} /[\mathrm{C} @] 4([\mathrm{H}]) \mathrm{C}[\mathrm{C} \\
@ @] 4(\mathrm{C}(\mathrm{O})=\mathrm{O}) \mathrm{NC}(=\mathrm{O})[\mathrm{C} @] 3([\mathrm{H}]) \mathrm{C} 1) \mathrm{NC}(=\mathrm{O}) \mathrm{OC} 1 \mathrm{CCCC} 1)=\mathrm{CC}(=\mathrm{N} 2) \mathrm{C} 1=\mathrm{CSC}(\mathrm{NC} \\
(\mathrm{C}) \mathrm{C})=\mathrm{N} 1\end{array}$ \\
\hline 35. & Clomethiazole & $\begin{array}{l}\text { Sedative and hypnotic, that is widely used in treating and } \\
\text { preventing symptoms of acute alcohol withdrawal }\end{array}$ & Yes & $\mathrm{CC} 1=\mathrm{C}(\mathrm{CCCl}) \mathrm{SC}=\mathrm{N} 1$ \\
\hline 36. & Cobicistat & Antiviral, booster of protein kinase inhibitors & Yes & $\begin{array}{l}\mathrm{CC}(\mathrm{C}) \mathrm{C} 1=\mathrm{NC}(\mathrm{CN}(\mathrm{C}) \mathrm{C}(=\mathrm{O}) \mathrm{N}[\mathrm{C} @ @ \mathrm{H}](\mathrm{CCN} 2 \mathrm{CCOCC} 2) \mathrm{C}(=\mathrm{O}) \mathrm{N}[\mathrm{C} @ \mathrm{H}](\mathrm{CC}[\mathrm{C} @ \mathrm{H}]( \\
\mathrm{CC} 2=\mathrm{CC}=\mathrm{CC}=\mathrm{C} 2) \mathrm{NC}(=\mathrm{O}) \mathrm{OCC} 2=\mathrm{CN}=\mathrm{CS} 2) \mathrm{CC} 2=\mathrm{CC}=\mathrm{CC}=\mathrm{C} 2)=\mathrm{CS} 1\end{array}$ \\
\hline 37. & Cocarboxylase & Coenzyme form of Vitamin B1 & Yes & $\mathrm{CC} 1=\mathrm{C}(\mathrm{CCO}[\mathrm{P} @](\mathrm{O})(=\mathrm{O}) \mathrm{O}[\mathrm{P} @](\mathrm{O})([\mathrm{O}-])=\mathrm{O}) \mathrm{SC}=[\mathrm{N}+] 1 \mathrm{CC} 1=\mathrm{CN}=\mathrm{C}(\mathrm{C}) \mathrm{N}=\mathrm{C} 1 \mathrm{~N}$ \\
\hline 38. & Dabrafenib & $\begin{array}{l}\text { Approved to treat anaplastic thyroid cancer caused by an } \\
\text { abnormal BRAF V600E gene }\end{array}$ & Yes & $\begin{array}{l}\mathrm{CC}(\mathrm{C})(\mathrm{C}) \mathrm{C} 1=\mathrm{NC}(=\mathrm{C}(\mathrm{S} 1) \mathrm{C} 1=\mathrm{NC}(\mathrm{N})=\mathrm{NC}=\mathrm{C} 1) \mathrm{C} 1=\mathrm{C}(\mathrm{F}) \mathrm{C}(\mathrm{NS}(=\mathrm{O})(=\mathrm{O}) \mathrm{C} 2=\mathrm{C}(\mathrm{F}) \mathrm{C}=\mathrm{CC} \\
=\mathrm{C} 2 \mathrm{~F})=\mathrm{CC}=\mathrm{C} 1\end{array}$ \\
\hline 39. & Dasatinib & $\begin{array}{l}\text { Approved for use in patients with Chronic } \\
\text { MyelogenousLeukemia (CML) }\end{array}$ & Yes & $\begin{array}{l}\mathrm{CC} 1=\mathrm{NC}(\mathrm{NC} 2=\mathrm{NC}=\mathrm{C}(\mathrm{S} 2) \mathrm{C}(=\mathrm{O}) \mathrm{NC} 2=\mathrm{C}(\mathrm{C}) \mathrm{C}=\mathrm{CC}=\mathrm{C} 2 \mathrm{Cl})=\mathrm{CC}(=\mathrm{N} 1) \mathrm{N} 1 \mathrm{CCN}(\mathrm{CCO}) \mathrm{C} \\
\mathrm{C} 1\end{array}$ \\
\hline
\end{tabular}




\begin{tabular}{|c|c|c|c|c|}
\hline 40. & Dexpramipexole & $\begin{array}{l}\text { Used for the treatment of Parkinson's disease and restless leg } \\
\text { syndrome }\end{array}$ & Yes & $\mathrm{CCCN}[\mathrm{C} @ @ \mathrm{H}] 1 \mathrm{CCC} 2=\mathrm{C}(\mathrm{C} 1) \mathrm{SC}(\mathrm{N})=\mathrm{N} 2$ \\
\hline 41. & Dimazole & Antifungal agent & Yes & $\mathrm{CCN}(\mathrm{CC}) \mathrm{CCOC} 1=\mathrm{CC} 2=\mathrm{C}(\mathrm{C}=\mathrm{C} 1) \mathrm{N}=\mathrm{C}(\mathrm{S} 2) \mathrm{N}(\mathrm{C}) \mathrm{C}$ \\
\hline 42. & Disperse Blue 106 & Allergen and dermatological sensitizer & Yes & $\mathrm{CCN}(\mathrm{CCO}) \mathrm{C} 1=\mathrm{CC}=\mathrm{C}(\mathrm{N}=\mathrm{NC} 2=\mathrm{NC}=\mathrm{C}(\mathrm{S} 2) \mathrm{N}(=\mathrm{O})=\mathrm{O}) \mathrm{C}(\mathrm{C})=\mathrm{C} 1$ \\
\hline 43. & Dolastatin 10 & $\begin{array}{l}\text { For the treatment of sarcoma, leukemia, lymphoma, liver } \\
\text { cancer, and kidney cancer }\end{array}$ & No & $\begin{array}{l}\mathrm{CC}[\mathrm{C} @ \mathrm{H}](\mathrm{C})[\mathrm{C} @ @ \mathrm{H}]([\mathrm{C} @ @ \mathrm{H}](\mathrm{CC}=\mathrm{O}) \mathrm{N} 1 \mathrm{CCC}[\mathrm{C} @ \mathrm{H}] 1[\mathrm{C} @ \mathrm{H}](\mathrm{OC})[\mathrm{C} @ @ \mathrm{H}](\mathrm{C}) \mathrm{C} \\
(=\mathrm{O}) \mathrm{N}[\mathrm{C} @ @ \mathrm{H}](\mathrm{CC} 1=\mathrm{CC}=\mathrm{CC}=\mathrm{C} 1) \mathrm{C} 1=\mathrm{NC}=\mathrm{CS} 1) \mathrm{OC}) \mathrm{N}(\mathrm{C}) \mathrm{C}(=\mathrm{O})[\mathrm{C} @ @ \mathrm{H}](\mathrm{NC}(=\mathrm{O})[ \\
\mathrm{C} @ \mathrm{H}](\mathrm{C}(\mathrm{C}) \mathrm{C}) \mathrm{N}(\mathrm{C}) \mathrm{C}) \mathrm{C}(\mathrm{C}) \mathrm{C}\end{array}$ \\
\hline 44. & Edoxaban & Treatment of stroke and systemic embolism & No & $\begin{array}{l}\mathrm{CN}(\mathrm{C}) \mathrm{C}(=\mathrm{O})[\mathrm{C} @ \mathrm{H}] 1 \mathrm{CC}[\mathrm{C} @ \mathrm{H}](\mathrm{NC}(=\mathrm{O}) \mathrm{C}(=\mathrm{O}) \mathrm{NC} 2=\mathrm{NC}=\mathrm{C}(\mathrm{Cl}) \mathrm{C}=\mathrm{C} 2)[\mathrm{C} @ @ \mathrm{H}](\mathrm{C} 1) \\
\mathrm{NC}(=\mathrm{O}) \mathrm{C} 1=\mathrm{NC} 2=\mathrm{C}(\mathrm{CN}(\mathrm{C}) \mathrm{CC} 2) \mathrm{S} 1\end{array}$ \\
\hline 45. & Epothilone D & $\begin{array}{l}\text { Investigated for treatment in colorectal cancer, lung cancer, } \\
\text { breast cancer, solid tumors, and prostate cancer }\end{array}$ & Yes & $\begin{array}{l}{[\mathrm{H}][\mathrm{C} @] 1(\mathrm{CC}=\mathrm{C}(\mathrm{C}) / \mathrm{CCC}[\mathrm{C} @ \mathrm{H}](\mathrm{C})[\mathrm{C} @ \mathrm{H}](\mathrm{O})[\mathrm{C} @ @ \mathrm{H}](\mathrm{C}) \mathrm{C}(=\mathrm{O}) \mathrm{C}(\mathrm{C})(\mathrm{C})[\mathrm{C} @ @ \mathrm{H}](} \\
\mathrm{O}) \mathrm{CC}(=\mathrm{O}) \mathrm{O} 1) \mathrm{C}(\mathrm{C})=\mathrm{CC} 1=\mathrm{CSC}(\mathrm{C})=\mathrm{N} 1\end{array}$ \\
\hline 46. & Ethoxzolamide & Used as diuretic and in glaucoma & Yes & $\mathrm{CCOC} 1=\mathrm{CC} 2=\mathrm{C}(\mathrm{C}=\mathrm{C} 1) \mathrm{N}=\mathrm{C}(\mathrm{S} 2) \mathrm{S}(\mathrm{N})(=\mathrm{O})=\mathrm{O}$ \\
\hline 47. & Faldaprevir & Investigated for the treatment of chronic Hepatitis C. & No & $\begin{array}{l}\mathrm{COC} 1=\mathrm{C}(\mathrm{Br}) \mathrm{C} 2=\mathrm{C}(\mathrm{C}=\mathrm{C} 1) \mathrm{C}(\mathrm{O}[\mathrm{C} @ @ \mathrm{H}] 1 \mathrm{C}[\mathrm{C} @ \mathrm{H}](\mathrm{N}(\mathrm{C} 1) \mathrm{C}(=\mathrm{O})[\mathrm{C} @ @ \mathrm{H}](\mathrm{NC}(=\mathrm{O}) \mathrm{O} \\
\mathrm{C} 1 \mathrm{CCCC} 1) \mathrm{C}(\mathrm{C})(\mathrm{C}) \mathrm{C}) \mathrm{C}(=\mathrm{O}) \mathrm{N}[\mathrm{C} @ @] 1(\mathrm{C}[\mathrm{C} @ \mathrm{H}] 1 \mathrm{C}=\mathrm{C}) \mathrm{C}(\mathrm{O})=\mathrm{O})=\mathrm{CC}(=\mathrm{N} 2) \mathrm{C} 1=\mathrm{CSC}( \\
\mathrm{NC}(=\mathrm{O}) \mathrm{C}(\mathrm{C}) \mathrm{C})=\mathrm{N} 1\end{array}$ \\
\hline 48. & Famotidine & $\begin{array}{l}\text { Commonly used in gastrointestinal conditions related to acid } \\
\text { secretion, such as gastric ulcers and Gastroesophageal Reflux } \\
\text { Disease (GERD) }\end{array}$ & Yes & $\mathrm{NC}(\mathrm{N})=\mathrm{NC} 1=\mathrm{NC}(\mathrm{CSCCC}(\mathrm{N})=\mathrm{NS}(\mathrm{N})(=\mathrm{O})=\mathrm{O})=\mathrm{CS} 1$ \\
\hline 49. & Febuxostat & Used for the treatment of hyperuricemia in patients with gout & Yes & $\mathrm{CC}(\mathrm{C}) \mathrm{COC} 1=\mathrm{C}(\mathrm{C}=\mathrm{C}(\mathrm{C}=\mathrm{C} 1) \mathrm{C} 1=\mathrm{NC}(\mathrm{C})=\mathrm{C}(\mathrm{S} 1) \mathrm{C}(\mathrm{O})=\mathrm{O}) \mathrm{C \# N}$ \\
\hline 50. & Flutemetamol & Used as a diagnostic tool for Alzheimer's disease & Yes & $\mathrm{CNC} 1=\mathrm{C}(\mathrm{F}) \mathrm{C}=\mathrm{C}(\mathrm{C}=\mathrm{C} 1) \mathrm{C} 1=\mathrm{NC} 2=\mathrm{C}(\mathrm{S} 1) \mathrm{C}=\mathrm{C}(\mathrm{O}) \mathrm{C}=\mathrm{C} 2$ \\
\hline 51. & Flutemetamol (18F) & A Positron Emission Tomography (PET) radiotracer & Yes & $\mathrm{CNC} 1=\mathrm{C}([18 \mathrm{~F}]) \mathrm{C}=\mathrm{C}(\mathrm{C}=\mathrm{C} 1) \mathrm{C} 1=\mathrm{NC} 2=\mathrm{CC}=\mathrm{C}(\mathrm{O}) \mathrm{C}=\mathrm{C} 2 \mathrm{~S} 1$ \\
\hline 52. & Fosravuconazole & Antifungal activity & No & $\begin{array}{l}\mathrm{C}[\mathrm{C} @ @ \mathrm{H}](\mathrm{C} 1=\mathrm{NC}(=\mathrm{CS} 1) \mathrm{C} 1=\mathrm{CC}=\mathrm{C}(\mathrm{C}=\mathrm{C} 1) \mathrm{C} \# \mathrm{~N})[\mathrm{C} @ @](\mathrm{CN} 1 \mathrm{C}=\mathrm{NC}=\mathrm{N} 1)(\mathrm{OCOP}(\mathrm{O} \\
)(\mathrm{O})=\mathrm{O}) \mathrm{C} 1=\mathrm{CC}=\mathrm{C}(\mathrm{F}) \mathrm{C}=\mathrm{C} 1 \mathrm{~F}\end{array}$ \\
\hline 53. & GDC-0917 & Treatment of lymphoma and solid cancers. & No & $\begin{array}{l}\mathrm{CN}[\mathrm{C} @ @ \mathrm{H}](\mathrm{C}) \mathrm{C}(=\mathrm{O}) \mathrm{N}[\mathrm{C} @ @ \mathrm{H}](\mathrm{C} 1 \mathrm{CCCCC} 1) \mathrm{C}(=\mathrm{O}) \mathrm{N} 1 \mathrm{CCC}[\mathrm{C} @ \mathrm{H}] 1 \mathrm{C}(=\mathrm{O}) \mathrm{NC} 1=\mathrm{C}( \\
\mathrm{N}=\mathrm{C}(\mathrm{S} 1) \mathrm{C} 1=\mathrm{NC}=\mathrm{CO} 1) \mathrm{C} 1=\mathrm{CC}=\mathrm{CC}=\mathrm{C} 1\end{array}$ \\
\hline 54. & GS-9256 & $\begin{array}{l}\text { Used in trials studying the treatment of } \mathrm{HCV} \text { infection and } \\
\text { chronic Hepatitis C infection }\end{array}$ & No & $\begin{array}{l}\mathrm{COC} 1=\mathrm{C}(\mathrm{Cl}) \mathrm{C} 2=\mathrm{NC}(=\mathrm{CC}(\mathrm{O}[\mathrm{C} @ @ \mathrm{H}] 3 \mathrm{C}[\mathrm{C} @ @ \mathrm{H}] 4 \mathrm{~N}(\mathrm{C} 3) \mathrm{C}(=\mathrm{O})[\mathrm{C} @ \mathrm{H}](\mathrm{CCCCCCC} \\
\mathrm{C} @ @ \mathrm{H}] 3 \mathrm{C}[\mathrm{C} @] 3(\mathrm{NC} 4=\mathrm{O}) \mathrm{P}(\mathrm{O})(=\mathrm{O}) \mathrm{CC} 3=\mathrm{C}(\mathrm{F}) \mathrm{C}=\mathrm{CC}=\mathrm{C} 3 \mathrm{~F}) \mathrm{NC}(=\mathrm{O}) \mathrm{OC} 3 \mathrm{CCCC} 3)=\mathrm{C} \\
2 \mathrm{C}=\mathrm{C} 1) \mathrm{C} 1=\mathrm{CSC}(\mathrm{NC}(\mathrm{C}) \mathrm{C})=\mathrm{N} 1\end{array}$ \\
\hline 55. & GSK-356278 & $\begin{array}{l}\text { A potent, selective, brain-penetrant phosphodiesterase- } 4 \\
\text { inhibitor }\end{array}$ & Yes & $\mathrm{CCN} 1 \mathrm{~N}=\mathrm{CC} 2=\mathrm{C}(\mathrm{NC} 3 \mathrm{CCOCC} 3) \mathrm{C}(=\mathrm{CN}=\mathrm{C} 12) \mathrm{C} 1=\mathrm{NN}=\mathrm{C}(\mathrm{CC} 2=\mathrm{C}(\mathrm{C}) \mathrm{N}=\mathrm{C}(\mathrm{C}) \mathrm{S} 2) \mathrm{O} 1$ \\
\hline 56. & Isatoribine & Used for the treatment of Hepatitis C & Yes & $\mathrm{NC} 1=\mathrm{NC}(=\mathrm{O}) \mathrm{C} 2=\mathrm{C}(\mathrm{N} 1) \mathrm{N}([\mathrm{C} @ @ \mathrm{H}] 1 \mathrm{O}[\mathrm{C} @ \mathrm{H}](\mathrm{CO})[\mathrm{C} @ @ \mathrm{H}](\mathrm{O})[\mathrm{C} @ \mathrm{H}] 1 \mathrm{O}) \mathrm{C}(=\mathrm{O}) \mathrm{S} 2$ \\
\hline 57. & Isavuconazole & Broad spectrum antifungal agent & Yes & $\begin{array}{l}\mathrm{C}[\mathrm{C} @ @ \mathrm{H}](\mathrm{C} 1=\mathrm{NC}(=\mathrm{CS} 1) \mathrm{C} 1=\mathrm{CC}=\mathrm{C}(\mathrm{C}=\mathrm{C} 1) \mathrm{C} \# \mathrm{~N})[\mathrm{C} @](\mathrm{O})(\mathrm{CN} 1 \mathrm{C}=\mathrm{NC}=\mathrm{N} 1) \mathrm{C} 1=\mathrm{C}(\mathrm{F}) \\
\mathrm{C}=\mathrm{CC}(\mathrm{F})=\mathrm{C} 1\end{array}$ \\
\hline 58. & Isavuconazonium & $\begin{array}{l}\text { Indicated in the treatment of invasive aspergillosis and } \\
\text { invasive mucormycosis infection }\end{array}$ & No & $\begin{array}{l}{[\mathrm{H}] \mathrm{C}(\mathrm{C})(\mathrm{OC}(=\mathrm{O}) \mathrm{N}(\mathrm{C}) \mathrm{C} 1=\mathrm{C}(\mathrm{COC}(=\mathrm{O}) \mathrm{CNC}) \mathrm{C}=\mathrm{CC}=\mathrm{N} 1)[\mathrm{N}+] 1=\mathrm{CN}(\mathrm{C}[\mathrm{C} @](\mathrm{O})(\mathrm{C} 2=\mathrm{C}} \\
(\mathrm{F}) \mathrm{C}=\mathrm{CC}(\mathrm{F})=\mathrm{C} 2)[\mathrm{C} @ @]([\mathrm{H}])(\mathrm{C}) \mathrm{C} 2=\mathrm{NC}(=\mathrm{CS} 2) \mathrm{C} 2=\mathrm{CC}=\mathrm{C}(\mathrm{C}=\mathrm{C} 2) \mathrm{C} \# \mathrm{~N}) \mathrm{N}=\mathrm{C} 1\end{array}$ \\
\hline 59. & Ixabepilone & Approved for the treatment of metastatic breast cancer & Yes & $\begin{array}{l}{[\mathrm{H}][\mathrm{C} @] 12 \mathrm{C}[\mathrm{C} @ \mathrm{H}](\mathrm{NC}(=\mathrm{O}) \mathrm{C}[\mathrm{C} @ \mathrm{H}](\mathrm{O}) \mathrm{C}(\mathrm{C})(\mathrm{C}) \mathrm{C}(=\mathrm{O})[\mathrm{C} @ \mathrm{H}](\mathrm{C})[\mathrm{C} @ @ \mathrm{H}](\mathrm{O})[\mathrm{C} @} \\
@, \mathrm{H}](\mathrm{C}) \mathrm{CCC}[\mathrm{C} @ @] 1(\mathrm{C}) \mathrm{O} 2) \mathrm{C}(\mathrm{C})=\mathrm{CC} 1=\mathrm{CSC}(\mathrm{C})=\mathrm{N} 1\end{array}$ \\
\hline 60. & JNJ-41443532 & $\begin{array}{l}\text { Has been used in trials for the treatment of diabetes mellitus, } \\
\text { type } 2\end{array}$ & Yes & $\begin{array}{l}\mathrm{O}[\mathrm{C} @] 1(\mathrm{CC}[\mathrm{C} @ @ \mathrm{H}](\mathrm{CC} 1) \mathrm{N} 1 \mathrm{CC}(\mathrm{C} 1) \mathrm{NC}(=\mathrm{O}) \mathrm{CNC}(=\mathrm{O}) \mathrm{C} 1=\mathrm{CC}=\mathrm{CC}(=\mathrm{C} 1) \mathrm{C}(\mathrm{F})(\mathrm{F}) \mathrm{F}) \\
\mathrm{C} 1=\mathrm{CN}=\mathrm{CS} 1\end{array}$ \\
\hline
\end{tabular}




\begin{tabular}{|c|c|c|c|c|}
\hline 61. & JTK-853 & Exhibited activity against $\mathrm{HCV}$ & No & $\begin{array}{l}\mathrm{FC} 1=\mathrm{C}(\mathrm{OC}(\mathrm{F})(\mathrm{F}) \mathrm{F}) \mathrm{C}=\mathrm{CC}(\mathrm{CNC}(=\mathrm{O})[\mathrm{C} @ \mathrm{H}] 2 \mathrm{CN}(\mathrm{CCN} 2 \mathrm{~S}(=\mathrm{O})(=\mathrm{O}) \mathrm{C} 2=\mathrm{CC}=\mathrm{C}(\mathrm{C}=\mathrm{C} 2) \\
\mathrm{C}(\mathrm{F})(\mathrm{F}) \mathrm{F}) \mathrm{C} 2=\mathrm{NC} 3=\mathrm{C}(\mathrm{S} 2) \mathrm{C}=\mathrm{NC}(=\mathrm{N} 3) \mathrm{C} 2 \mathrm{CC} 2)=\mathrm{C} 1\end{array}$ \\
\hline 62. & KOS-1584 & $\begin{array}{l}\text { Anticancer agents that have demonstrated activity in taxane- } \\
\text { resistant tumors. Kos- } 1584\end{array}$ & Yes & $\begin{array}{l}\mathrm{C}[\mathrm{C} @ \mathrm{H}] 1 \mathrm{C}=\mathrm{CCC}(\mathrm{C})=\mathrm{C} / \mathrm{C}[\mathrm{C} @ \mathrm{H}](\mathrm{OC}(=\mathrm{O}) \mathrm{C}[\mathrm{C} @ \mathrm{H}](\mathrm{O}) \mathrm{C}(\mathrm{C})(\mathrm{C}) \mathrm{C}(=\mathrm{O})[\mathrm{C} @ \mathrm{H}](\mathrm{C})[\mathrm{C} @ \\
\mathrm{H}] 1 \mathrm{O}) \mathrm{C}(\mathrm{C})=\mathrm{CC} 1=\mathrm{CSC}(\mathrm{C})=\mathrm{N} 1\end{array}$ \\
\hline 63. & LCL-161 & $\begin{array}{l}\text { In trials for the treatment of leukemia, neoplasms, solid } \\
\text { tumors, breast cancer, and ovarian cancer, among others }\end{array}$ & Yes & $\begin{array}{l}\mathrm{CN}[\mathrm{C} @ @ \mathrm{H}](\mathrm{C}) \mathrm{C}(=\mathrm{O}) \mathrm{N}[\mathrm{C} @ @ \mathrm{H}](\mathrm{C} 1 \mathrm{CCCCC} 1) \mathrm{C}(=\mathrm{O}) \mathrm{N} 1 \mathrm{CCC}[\mathrm{C} @ \mathrm{H}] 1 \mathrm{C} 1=\mathrm{NC}(=\mathrm{CS} 1) \\
\mathrm{C}(=\mathrm{O}) \mathrm{C} 1=\mathrm{CC}=\mathrm{C}(\mathrm{F}) \mathrm{C}=\mathrm{C} 1\end{array}$ \\
\hline 64. & LFF-571 & $\begin{array}{l}\text { Used in trials studying the treatment of moderate clostridium } \\
\text { difficile infection }\end{array}$ & No & $\begin{array}{l}\mathrm{CNC}(=\mathrm{O}) \mathrm{C}[\mathrm{C} @ @ \mathrm{H}] 1 \mathrm{NC}(=\mathrm{O}) \mathrm{C} 2=\mathrm{CSC}(=\mathrm{N} 2) \mathrm{C} 2=\mathrm{CC}=\mathrm{C}(\mathrm{N}=\mathrm{C} 2 \mathrm{C} 2=\mathrm{CSC}(=\mathrm{N} 2) \mathrm{C} 2=\mathrm{CS} \\
\mathrm{C}(=\mathrm{N} 2)[\mathrm{C} @ @ \mathrm{H}](\mathrm{NC}(=\mathrm{O}) \mathrm{CNC}(=\mathrm{O}) \mathrm{C} 2=\mathrm{C}(\mathrm{COC}) \mathrm{SC}(=\mathrm{N} 2)[\mathrm{C} @ @ \mathrm{H}](\mathrm{NC}(=\mathrm{O}) \mathrm{C} 2=\mathrm{C}(\mathrm{C}) \\
\mathrm{SC} 1=\mathrm{N} 2) \mathrm{C}(\mathrm{C}) \mathrm{C})[\mathrm{C} @ @ \mathrm{H}](\mathrm{O}) \mathrm{C} 1=\mathrm{CC}=\mathrm{CC}=\mathrm{C} 1) \mathrm{C} 1=\mathrm{NC}(=\mathrm{CS} 1) \mathrm{N}(\mathrm{CCCC}(\mathrm{O})=\mathrm{O}) \mathrm{C}(=\mathrm{O} \\
\mathrm{O}[\mathrm{C} @ \mathrm{H}] 1 \mathrm{CC}[\mathrm{C} @ @ \mathrm{H}](\mathrm{CC} 1) \mathrm{C}(\mathrm{O})=\mathrm{O}\end{array}$ \\
\hline 65. & LY-2608204 & Treatment of type- 2 diabetes & No & $\begin{array}{l}\mathrm{O}=\mathrm{C}(\mathrm{NC} 1=\mathrm{NC}=\mathrm{C}(\mathrm{SCCN} 2 \mathrm{CCCC} 2) \mathrm{S} 1)[\mathrm{C} @ @] 1(\mathrm{C}[\mathrm{C} @ \mathrm{H}] 1 \mathrm{C} 1 \mathrm{CCCCC} 1) \mathrm{C} 1=\mathrm{CC}=\mathrm{C}(\mathrm{C} \\
=\mathrm{C} 1) \mathrm{S}(=\mathrm{O})(=\mathrm{O}) \mathrm{C} 1 \mathrm{CC} 1\end{array}$ \\
\hline 66. & Lanifibranor & Antifibrotic agent & Yes & $\mathrm{OC}(=\mathrm{O}) \mathrm{CCCC} 1=\mathrm{CC} 2=\mathrm{C}(\mathrm{C}=\mathrm{CC}(\mathrm{Cl})=\mathrm{C} 2) \mathrm{N} 1 \mathrm{~S}(=\mathrm{O})(=\mathrm{O}) \mathrm{C} 1=\mathrm{CC} 2=\mathrm{C}(\mathrm{C}=\mathrm{C} 1) \mathrm{N}=\mathrm{CS} 2$ \\
\hline 67. & Lintitript & $\begin{array}{l}\text { Used for the treatment of pancreatic cancer and appetite } \\
\text { disorders }\end{array}$ & Yes & $\mathrm{OC}(=\mathrm{O}) \mathrm{CN} 1 \mathrm{C}(=\mathrm{CC} 2=\mathrm{CC}=\mathrm{CC}=\mathrm{C} 12) \mathrm{C}(=\mathrm{O}) \mathrm{NC} 1=\mathrm{NC}(=\mathrm{CS} 1) \mathrm{C} 1=\mathrm{CC}=\mathrm{CC}=\mathrm{C} 1 \mathrm{Cl}$ \\
\hline 68. & Lusutrombopag & Treatment of thrombocytopenia & No & $\begin{array}{l}\mathrm{CCCCCCO}[\mathrm{C} @ @ \mathrm{H}](\mathrm{C}) \mathrm{C} 1=\mathrm{C}(\mathrm{OC}) \mathrm{C}(=\mathrm{CC}=\mathrm{C} 1) \mathrm{C} 1=\mathrm{CSC}(\mathrm{NC}(=\mathrm{O}) \mathrm{C} 2=\mathrm{CC}(\mathrm{Cl})=\mathrm{C}(\mathrm{C}=\mathrm{C} \\
(/ \mathrm{C}) \mathrm{C}(\mathrm{O})=\mathrm{O}) \mathrm{C}(\mathrm{Cl})=\mathrm{C} 2)=\mathrm{N} 1\end{array}$ \\
\hline 69. & MB-07803 & Treatment of type-2 diabetes & No & $\begin{array}{l}\mathrm{CCOC}(=\mathrm{O}) \mathrm{C}(\mathrm{C})(\mathrm{C}) \mathrm{NP}(=\mathrm{O})(\mathrm{NC}(\mathrm{C})(\mathrm{C}) \mathrm{C}(=\mathrm{O}) \mathrm{OCC}) \mathrm{C} 1=\mathrm{CC}=\mathrm{C}(\mathrm{O} 1) \mathrm{C} 1=\mathrm{C}(\mathrm{SC}(\mathrm{N})=\mathrm{N} 1) \mathrm{C} \\
(=\mathrm{O}) \mathrm{C}(\mathrm{C})(\mathrm{C}) \mathrm{C}\end{array}$ \\
\hline 70. & MBX-2982 & Has been used in trials studying the treatment of diabetes & Yes & $\begin{array}{l}\mathrm{CCC} 1=\mathrm{CN}=\mathrm{C}(\mathrm{N}=\mathrm{C} 1) \mathrm{N} 1 \mathrm{CCC}(\mathrm{CC} 1) \mathrm{C} 1=\mathrm{NC}(\mathrm{COC} 2=\mathrm{CC}=\mathrm{C}(\mathrm{C}=\mathrm{C} 2) \mathrm{N} 2 \mathrm{C}=\mathrm{NN}=\mathrm{N} 2)=\mathrm{CS} \\
1\end{array}$ \\
\hline 71. & MK-5108 & Aurora -A kinase inhibitor, antitumor activity & Yes & $\begin{array}{l}\mathrm{OC}(=\mathrm{O})[\mathrm{C} @ @] 1(\mathrm{CC} 2=\mathrm{CC}=\mathrm{CC}(\mathrm{NC} 3=\mathrm{NC}=\mathrm{CS} 3)=\mathrm{N} 2) \mathrm{CC}[\mathrm{C} @ @ \mathrm{H}](\mathrm{CC} 1) \mathrm{OC} 1=\mathrm{C}(\mathrm{F}) \mathrm{C}( \\
\mathrm{Cl})=\mathrm{CC}=\mathrm{C} 1\end{array}$ \\
\hline 72. & Managlinatdialanetil & Used for the treatment of type 2 diabetes & Yes & $\begin{array}{l}{[\mathrm{H}][\mathrm{C} @ @](\mathrm{C})(\mathrm{NP}(=\mathrm{O})(\mathrm{N}[\mathrm{C} @ @]([\mathrm{H}])(\mathrm{C}) \mathrm{C}(=\mathrm{O}) \mathrm{OCC}) \mathrm{C} 1=\mathrm{CC}=\mathrm{C}(\mathrm{O} 1) \mathrm{C} 1=\mathrm{C}(\mathrm{CC}(\mathrm{C}) \mathrm{C})} \\
\mathrm{SC}(=\mathrm{N}) \mathrm{N} 1) \mathrm{C}(=\mathrm{O}) \mathrm{OCC}\end{array}$ \\
\hline 73. & Masitinib & $\begin{array}{l}\text { Tyrosine-kinase inhibitor used in the treatment of mast cell } \\
\text { tumors }\end{array}$ & Yes & $\begin{array}{l}\mathrm{CN} 1 \mathrm{CCN}(\mathrm{CC} 2=\mathrm{CC}=\mathrm{C}(\mathrm{C}=\mathrm{C} 2) \mathrm{C}(=\mathrm{O}) \mathrm{NC} 2=\mathrm{CC}(\mathrm{N}=\mathrm{C} 3 \mathrm{NC}(=\mathrm{CS} 3) \mathrm{C} 3=\mathrm{CN}=\mathrm{CC}=\mathrm{C} 3)=\mathrm{C}( \\
\mathrm{C}) \mathrm{C}=\mathrm{C} 2) \mathrm{CC} 1\end{array}$ \\
\hline 74. & Meloxicam & $\begin{array}{l}\text { Indicated for the symptomatic treatment of arthritis and } \\
\text { osteoarthritis }\end{array}$ & Yes & $\mathrm{CN} 1 \mathrm{C}(\mathrm{C}(=\mathrm{O}) \mathrm{NC} 2=\mathrm{NC}=\mathrm{C}(\mathrm{C}) \mathrm{S} 2)=\mathrm{C}(\mathrm{O}) \mathrm{C} 2=\mathrm{C}(\mathrm{C}=\mathrm{CC}=\mathrm{C} 2) \mathrm{S} 1(=\mathrm{O})=\mathrm{O}$ \\
\hline 75. & Mirabegron & Used for the management of overactive bladder & Yes & $\mathrm{NC} 1=\mathrm{NC}(\mathrm{CC}(=\mathrm{O}) \mathrm{NC} 2=\mathrm{CC}=\mathrm{C}(\mathrm{CCNC}[\mathrm{C} @ \mathrm{H}](\mathrm{O}) \mathrm{C} 3=\mathrm{CC}=\mathrm{CC}=\mathrm{C} 3) \mathrm{C}=\mathrm{C} 2)=\mathrm{CS} 1$ \\
\hline 76. & $\begin{array}{l}\text { Morpholinylmercaptoben } \\
\text { zothiazole }\end{array}$ & Used in the diagnosis of contact dermatitis & Yes & $\mathrm{C} 1 \mathrm{CN}(\mathrm{CCO} 1) \mathrm{SC} 1=\mathrm{NC} 2=\mathrm{CC}=\mathrm{CC}=\mathrm{C} 2 \mathrm{~S} 1$ \\
\hline 77. & Neladenosonbialanate & Investigated for the treatment of heart failure & No & $\begin{array}{l}\mathrm{C}[\mathrm{C} @ \mathrm{H}](\mathrm{N}) \mathrm{C}(=\mathrm{O}) \mathrm{N}[\mathrm{C} @ @ \mathrm{H}](\mathrm{C}) \mathrm{C}(=\mathrm{O}) \mathrm{OCCOC} 1=\mathrm{CC}=\mathrm{C}(\mathrm{C}=\mathrm{C} 1) \mathrm{C} 1=\mathrm{C}(\mathrm{C} \# \mathrm{~N}) \mathrm{C}(=\mathrm{NC}( \\
\mathrm{SCC} 2=\mathrm{CSC}(=\mathrm{N} 2) \mathrm{C} 2=\mathrm{CC}=\mathrm{C}(\mathrm{Cl}) \mathrm{C}=\mathrm{C} 2)=\mathrm{C} 1 \mathrm{C} \# \mathrm{~N}) \mathrm{N} 1 \mathrm{CCCC} 1\end{array}$ \\
\hline 78. & Nitazoxanide & Used for the treatment of diarrhoea in adults and children & Yes & $\mathrm{CC}(=\mathrm{O}) \mathrm{OC} 1=\mathrm{CC}=\mathrm{CC}=\mathrm{C} 1 \mathrm{C}(=\mathrm{O}) \mathrm{NC} 1=\mathrm{NC}=\mathrm{C}(\mathrm{S} 1)[\mathrm{N}+]([\mathrm{O}-])=\mathrm{O}$ \\
\hline 79. & Nizatidine & Used for the treatment of duodenal ulcers & Yes & $\mathrm{CNC}(\mathrm{NCCSCC} 1=\mathrm{CSC}(\mathrm{CN}(\mathrm{C}) \mathrm{C})=\mathrm{N} 1)=\mathrm{C}[\mathrm{N}+]([\mathrm{O}-])=\mathrm{O}$ \\
\hline 80. & ONO-8539 & Under investigation for oesophageal pain hypersensitivity & Yes & $\begin{array}{l}\mathrm{CC}(\mathrm{C}) \mathrm{CN}(\mathrm{C} 1=\mathrm{CC} 2=\mathrm{C}(\mathrm{CCC} 2) \mathrm{C}=\mathrm{C} 1 \mathrm{OCC} 1=\mathrm{CC}=\mathrm{C}(\mathrm{C}=\mathrm{C} 1 \mathrm{C}) \mathrm{C}(\mathrm{O})=\mathrm{O}) \mathrm{S}(=\mathrm{O})(=\mathrm{O}) \mathrm{C} 1=\mathrm{N} \\
\mathrm{C}=\mathrm{CS} 1\end{array}$ \\
\hline 81. & Oprozomib & Oral proteasome inhibitor with anti-tumor activity & No & $\begin{array}{l}\mathrm{COC}[\mathrm{C} @ \mathrm{H}](\mathrm{NC}(=\mathrm{O})[\mathrm{C} @ \mathrm{H}](\mathrm{COC}) \mathrm{NC}(=\mathrm{O}) \mathrm{C} 1=\mathrm{CN}=\mathrm{C}(\mathrm{C}) \mathrm{S} 1) \mathrm{C}(=\mathrm{O}) \mathrm{N}[\mathrm{C} @ @ \mathrm{H}](\mathrm{CC} 1= \\
\mathrm{CC}=\mathrm{CC}=\mathrm{C} 1) \mathrm{C}(=\mathrm{O})[\mathrm{C} @ @] 1(\mathrm{C}) \mathrm{CO} 1\end{array}$ \\
\hline 82. & PF-05089771 & Under investigation in patients with osteoarthritis of the knee & Yes & $\begin{array}{l}\mathrm{NC} 1=\mathrm{C}(\mathrm{C}=\mathrm{NN} 1) \mathrm{C} 1=\mathrm{CC}(\mathrm{Cl})=\mathrm{CC}=\mathrm{C} 1 \mathrm{OC} 1=\mathrm{CC}(\mathrm{F})=\mathrm{C}(\mathrm{C}=\mathrm{C} 1 \mathrm{Cl}) \mathrm{S}(=\mathrm{O})(=\mathrm{O}) \mathrm{NC} 1=\mathrm{CSC}= \\
\mathrm{N} 1\end{array}$ \\
\hline
\end{tabular}




\begin{tabular}{|c|c|c|c|c|}
\hline 83. & PF-06751979 & BACE1 inhibitor for Alzheimer's disease & Yes & $\begin{array}{l}\mathrm{CC} 1=\mathrm{CN} 2 \mathrm{C}=\mathrm{C}(\mathrm{CC}(=\mathrm{O}) \mathrm{N} 3 \mathrm{CCC} 4(\mathrm{CN}(\mathrm{C} 4)[\mathrm{C} @ @ \mathrm{H}] 4 \mathrm{CCC} 5=\mathrm{C} 4 \mathrm{C}=\mathrm{CC}(=\mathrm{C} 5) \mathrm{C} 4=\mathrm{CC}(\mathrm{C} \\
)=\mathrm{NC}=\mathrm{N} 4) \mathrm{CC} 3) \mathrm{N}=\mathrm{C} 2 \mathrm{~S} 1\end{array}$ \\
\hline 84. & PF-5190457 & Under investigation, novel ghrelin receptor inverse agonist & Yes & $\begin{array}{l}\mathrm{C}[\mathrm{C} @ \mathrm{H}] 1 \mathrm{C}[\mathrm{C} @ \mathrm{H}] 2 \mathrm{CSC}(\mathrm{N})=\mathrm{N}[\mathrm{C} @] 2(\mathrm{CO} 1) \mathrm{C} 1=\mathrm{NC}(\mathrm{NC}(=\mathrm{O}) \mathrm{C} 2=\mathrm{NC}=\mathrm{C}(\mathrm{OC}(\mathrm{F}) \mathrm{F}) \mathrm{C}= \\
\mathrm{C} 2)=\mathrm{CS} 1\end{array}$ \\
\hline 85. & Phthalylsulfathiazole & $\begin{array}{l}\text { Used as an antibiotic for bowel surgery, and for infections of } \\
\text { the colon }\end{array}$ & Yes & $\mathrm{OC}(=\mathrm{O}) \mathrm{C} 1=\mathrm{CC}=\mathrm{CC}=\mathrm{C} 1 \mathrm{C}(=\mathrm{O}) \mathrm{NC} 1=\mathrm{CC}=\mathrm{C}(\mathrm{C}=\mathrm{C} 1) \mathrm{S}(=\mathrm{O})(=\mathrm{O}) \mathrm{NC} 1=\mathrm{NC}=\mathrm{CS} 1$ \\
\hline 86. & Pittsburgh Compound B & $\begin{array}{l}\text { Radioactive analog of thioflavin } \mathrm{T} \text {, which can be used in } \\
\text { positron emission tomography scans to image beta-amyloid } \\
\text { plaques in neuronal tissue }\end{array}$ & Yes & {$[11 \mathrm{CH} 3] \mathrm{NC} 1=\mathrm{CC}=\mathrm{C}(\mathrm{C}=\mathrm{C} 1) \mathrm{C} 1=\mathrm{NC} 2=\mathrm{C}(\mathrm{S} 1) \mathrm{C}=\mathrm{C}(\mathrm{O}) \mathrm{C}=\mathrm{C} 2$} \\
\hline 87. & Pramipexole & Used to treat the symptoms of Parkinson's Disease & Yes & $\mathrm{CCCN}[\mathrm{C} @ \mathrm{H}] 1 \mathrm{CCC} 2=\mathrm{C}(\mathrm{C} 1) \mathrm{SC}(\mathrm{N})=\mathrm{N} 2$ \\
\hline 88. & Pritelivir & Used in trials for prevention of HSV-2 and genital herpes & Yes & $\mathrm{CN}(\mathrm{C}(=\mathrm{O}) \mathrm{CC} 1=\mathrm{CC}=\mathrm{C}(\mathrm{C}=\mathrm{C} 1) \mathrm{C} 1=\mathrm{CC}=\mathrm{CC}=\mathrm{N} 1) \mathrm{C} 1=\mathrm{NC}(\mathrm{C})=\mathrm{C}(\mathrm{S} 1) \mathrm{S}(\mathrm{N})(=\mathrm{O})=\mathrm{O}$ \\
\hline 89. & Quizartinib & Anti-leukemic activity & No & $\begin{array}{l}\mathrm{CC}(\mathrm{C})(\mathrm{C}) \mathrm{C} 1=\mathrm{CC}(\mathrm{NC}(=\mathrm{O}) \mathrm{NC} 2=\mathrm{CC}=\mathrm{C}(\mathrm{C}=\mathrm{C} 2) \mathrm{C} 2=\mathrm{CN} 3 \mathrm{C}(\mathrm{SC} 4=\mathrm{C} 3 \mathrm{C}=\mathrm{CC}(\mathrm{OCCN} 3 \mathrm{CC} \\
\mathrm{OCC} 3)=\mathrm{C} 4)=\mathrm{N} 2)=\mathrm{NO} 1\end{array}$ \\
\hline 90. & Ravuconazole & $\begin{array}{l}\text { Investigated for use/treatment in fungal infections, } \\
\text { aspergillosis, candidiasis, and onychomycosis }\end{array}$ & Yes & $\begin{array}{l}\mathrm{C}[\mathrm{C} @ @ \mathrm{H}](\mathrm{C} 1=\mathrm{NC}(=\mathrm{CS} 1) \mathrm{C} 1=\mathrm{CC}=\mathrm{C}(\mathrm{C}=\mathrm{C} 1) \mathrm{C} \# \mathrm{~N})[\mathrm{C} @](\mathrm{O})(\mathrm{CN} 1 \mathrm{C}=\mathrm{NC}=\mathrm{N} 1) \mathrm{C} 1=\mathrm{CC}= \\
\mathrm{C}(\mathrm{F}) \mathrm{C}=\mathrm{C} 1 \mathrm{~F}\end{array}$ \\
\hline 91. & Razupenem & The treatment of skin infections & Yes & $\begin{array}{l}\mathrm{C}[\mathrm{C} @ @ \mathrm{H}](\mathrm{O})[\mathrm{C} @ @ \mathrm{H}] 1[\mathrm{C} @ \mathrm{H}] 2[\mathrm{C} @ @ \mathrm{H}](\mathrm{C}) \mathrm{C}(\mathrm{SC} 3=\mathrm{NC}(=\mathrm{CS} 3) \mathrm{C} 3=\mathrm{C}[\mathrm{C} @ \mathrm{H}](\mathrm{C}) \mathrm{NC} \\
3)=\mathrm{C}(\mathrm{N} 2 \mathrm{C} 1=\mathrm{O}) \mathrm{C}(\mathrm{O})=\mathrm{O}\end{array}$ \\
\hline 92. & Riluzole & $\begin{array}{l}\text { Used as an anticonvulsant (anticonvulsants) and to prolong } \\
\text { the survival of patients with amyotrophic lateral sclerosis }\end{array}$ & Yes & $\mathrm{NC} 1=\mathrm{NC} 2=\mathrm{C}(\mathrm{S} 1) \mathrm{C}=\mathrm{C}(\mathrm{OC}(\mathrm{F})(\mathrm{F}) \mathrm{F}) \mathrm{C}=\mathrm{C} 2$ \\
\hline 93. & Ritanserin & Treatment of schizophrenia & Yes & $\begin{array}{l}\mathrm{CC} 1=\mathrm{C}(\mathrm{CCN} 2 \mathrm{CCC}(\mathrm{CC} 2)=\mathrm{C}(\mathrm{C} 2=\mathrm{CC}=\mathrm{C}(\mathrm{F}) \mathrm{C}=\mathrm{C} 2) \mathrm{C} 2=\mathrm{CC}=\mathrm{C}(\mathrm{F}) \mathrm{C}=\mathrm{C} 2) \mathrm{C}(=\mathrm{O}) \mathrm{N} 2 \mathrm{C}=\mathrm{CS} \\
\mathrm{C} 2=\mathrm{N} 1\end{array}$ \\
\hline 94. & Ritonavir & Antiviral, used for the treatment of HIV infection & No & $\begin{array}{l}\mathrm{CC}(\mathrm{C})[\mathrm{C} @ \mathrm{H}](\mathrm{NC}=\mathrm{O}) \mathrm{N}(\mathrm{C}) \mathrm{CC} 1=\mathrm{CSC}(=\mathrm{N} 1) \mathrm{C}(\mathrm{C}) \mathrm{C}) \mathrm{C}(=\mathrm{O}) \mathrm{N}[\mathrm{C} @ \mathrm{H}](\mathrm{C}[\mathrm{C} @ \mathrm{H}](\mathrm{O})[\mathrm{C} @ \\
\mathrm{H}](\mathrm{CC} 1=\mathrm{CC}=\mathrm{CC}=\mathrm{C} 1) \mathrm{NC}(=\mathrm{O}) \mathrm{OCC} 1=\mathrm{CN}=\mathrm{CS} 1) \mathrm{CC} 1=\mathrm{CC}=\mathrm{CC}=\mathrm{C} 1\end{array}$ \\
\hline 95. & Rovatirelin & $\begin{array}{l}\text { Under investigation in patients with spinocerebellar } \\
\text { degeneration }\end{array}$ & Yes & $\begin{array}{l}\text { C[C@@ } @ \text { H]1CCCN1C }(=\mathrm{O})[\mathrm{C} @ \mathrm{H}](\mathrm{CC} 1=\mathrm{CSC}=\mathrm{N} 1) \mathrm{NC}(=\mathrm{O})[\mathrm{C} @ \mathrm{H}] 1 \mathrm{NC}(=\mathrm{O}) \mathrm{O}[\mathrm{C} @ \mathrm{H}] 1 \\
\mathrm{C}\end{array}$ \\
\hline 96. & Ruzasvir & Used in trials studying the treatment of Hepatitis $\mathrm{C}$ infection & No & $\begin{array}{l}\mathrm{COC}(=\mathrm{O}) \mathrm{N}[\mathrm{C} @ @ \mathrm{H}](\mathrm{C}(\mathrm{C}) \mathrm{C}) \mathrm{C}(=\mathrm{O}) \mathrm{N} 1 \mathrm{CCC}[\mathrm{C} @ \mathrm{H}] 1 \mathrm{C} 1=\mathrm{NC}=\mathrm{C}(\mathrm{N} 1) \mathrm{C} 1=\mathrm{CC}=\mathrm{C} 2 \mathrm{~N} 3[\mathrm{C} \\
@ @ \mathrm{H}](\mathrm{OC} 4=\mathrm{C}(\mathrm{C} 3=\mathrm{CC} 2=\mathrm{C} 1) \mathrm{C}(\mathrm{F})=\mathrm{CC}(=\mathrm{C} 4) \mathrm{C} 1=\mathrm{CN}=\mathrm{C}(\mathrm{N} 1)[\mathrm{C} @ @ \mathrm{H}] 1 \mathrm{CCCN} 1 \mathrm{C}(=\mathrm{O}) \\
[\mathrm{C} @ @ \mathrm{H}](\mathrm{NC}(=\mathrm{O}) \mathrm{OC}) \mathrm{C}(\mathrm{C}) \mathrm{C}) \mathrm{C} 1=\mathrm{CN}=\mathrm{C}(\mathrm{S} 1) \mathrm{C} 1 \mathrm{CC} 1\end{array}$ \\
\hline 97. & SAR-125844 & Anti-tumor activity & No & $\begin{array}{l}\mathrm{FC} 1=\mathrm{CC}=\mathrm{C}(\mathrm{C}=\mathrm{C} 1) \mathrm{C} 1=\mathrm{NN} 2 \mathrm{C}(\mathrm{SC} 3=\mathrm{CC}=\mathrm{C} 4 \mathrm{~N}=\mathrm{C}(\mathrm{NC}(=\mathrm{O}) \mathrm{NCCN} 5 \mathrm{CCOCC} 5) \mathrm{SC} 4=\mathrm{C} 3) \\
=\mathrm{NN}=\mathrm{C} 2 \mathrm{C}=\mathrm{C} 1\end{array}$ \\
\hline 98. & SB-649868 & Treatment of insomnia & Yes & $\begin{array}{l}\mathrm{CC} 1=\mathrm{NC}(\mathrm{C}(=\mathrm{O}) \mathrm{N} 2 \mathrm{CCCC}[\mathrm{C} @ \mathrm{H}] 2 \mathrm{CNC}(=\mathrm{O}) \mathrm{C} 2=\mathrm{CC}=\mathrm{CC} 3=\mathrm{C} 2 \mathrm{C}=\mathrm{CO} 3)=\mathrm{C}(\mathrm{S} 1) \mathrm{C} 1=\mathrm{CC} \\
=\mathrm{C}(\mathrm{F}) \mathrm{C}=\mathrm{C} 1\end{array}$ \\
\hline 99. & SNS-032 & $\begin{array}{l}\text { Investigated for use/treatment in cancer/tumors (unspecified), } \\
\text { leukemia (unspecified), lymphoma (unspecified), multiple } \\
\text { myeloma, and solid tumors }\end{array}$ & Yes & $\mathrm{CC}(\mathrm{C})(\mathrm{C}) \mathrm{C} 1=\mathrm{CN}=\mathrm{C}(\mathrm{CSC} 2=\mathrm{CN}=\mathrm{C}(\mathrm{NC}(=\mathrm{O}) \mathrm{C} 3 \mathrm{CCNCC} 3) \mathrm{S} 2) \mathrm{O} 1$ \\
\hline 100. & SNS-314 & $\begin{array}{l}\text { Potent and selective inhibitor of Aurora kinases A, B, and C, } \\
\text { anti-tumor agent }\end{array}$ & Yes & $\mathrm{ClC} 1=\mathrm{CC}=\mathrm{CC}(\mathrm{NC}(=\mathrm{O}) \mathrm{NC} 2=\mathrm{NC}=\mathrm{C}(\mathrm{CCNC} 3=\mathrm{C} 4 \mathrm{SC}=\mathrm{CC} 4=\mathrm{NC}=\mathrm{N} 3) \mathrm{S} 2)=\mathrm{C} 1$ \\
\hline 101. & SRT-2104 & $\begin{array}{l}\text { Treatment of sepsis, psoriasis, atrophy and diabetes mellitus, } \\
\text { Type } 2\end{array}$ & Yes & $\begin{array}{l}\mathrm{CC} 1=\mathrm{C}(\mathrm{SC}(=\mathrm{N} 1) \mathrm{C} 1=\mathrm{CC}=\mathrm{CN}=\mathrm{C} 1) \mathrm{C}(=\mathrm{O}) \mathrm{NC} 1=\mathrm{CC}=\mathrm{CC}=\mathrm{C} 1 \mathrm{C} 1=\mathrm{CN} 2 \mathrm{C}(\mathrm{CN} 3 \mathrm{CCOCC} 3) \\
=\mathrm{CSC} 2=\mathrm{N} 1\end{array}$ \\
\hline 102. & Sagopilone & $\begin{array}{l}\text { used in trials studying the treatment of melanoma, neoplasms, } \\
\text { CNS disease, breast cancer, and breast neoplasms }\end{array}$ & No & $\begin{array}{l}\mathrm{C}[\mathrm{C} @ \mathrm{H}] 1 \mathrm{CCC}[\mathrm{C} @ @] 2(\mathrm{C}) \mathrm{O}[\mathrm{C} @ \mathrm{H}] 2 \mathrm{C}[\mathrm{C} @ \mathrm{H}](\mathrm{OC}(=\mathrm{O}) \mathrm{C}[\mathrm{C} @ \mathrm{H}](\mathrm{O}) \mathrm{C}(\mathrm{C})(\mathrm{C}) \mathrm{C}(=\mathrm{O})[\mathrm{C} \\
@ \mathrm{H}](\mathrm{CC}=\mathrm{C})[\mathrm{C} @ \mathrm{H}] 1 \mathrm{O}) \mathrm{C}=\mathrm{CC}=\mathrm{C} 2 \mathrm{SC}(\mathrm{C})=\mathrm{NC} 2=\mathrm{C} 1\end{array}$ \\
\hline 103. & Simeprevir & $\begin{array}{l}\text { Indicated for the treatment of adults with chronic } \mathrm{HCV} \\
\text { infection }\end{array}$ & No & $\begin{array}{l}{[\mathrm{H}] \mathrm{C} 1=\mathrm{C}([\mathrm{H}])[\mathrm{C} @] 2([\mathrm{H}]) \mathrm{C}[\mathrm{C} @] 2(\mathrm{~N}=\mathrm{C}(\mathrm{O})[\mathrm{C} @] 2([\mathrm{H}]) \mathrm{C}[\mathrm{C} @]([\mathrm{H}])(\mathrm{C}[\mathrm{C} @ @] 2([\mathrm{H}]) \mathrm{C}} \\
(=\mathrm{O}) \mathrm{N}(\mathrm{C}) \mathrm{CCCC} 1) \mathrm{OC} 1=\mathrm{C} 2 \mathrm{C}=\mathrm{CC}(\mathrm{OC})=\mathrm{C}(\mathrm{C}) \mathrm{C} 2=\mathrm{NC}(=\mathrm{C} 1) \mathrm{C} 1=\mathrm{NC}(=\mathrm{CS} 1) \mathrm{C}(\mathrm{C}) \mathrm{C}) \mathrm{C}(\mathrm{O}) \\
=\mathrm{NS}(=\mathrm{O})(=\mathrm{O}) \mathrm{C} 1 \mathrm{CC} 1\end{array}$ \\
\hline
\end{tabular}




\begin{tabular}{|c|c|c|c|c|}
\hline 104. & Sudoxicam & nonsteroidal anti-inflammatory drugs & Yes & $\mathrm{CN} 1 \mathrm{C}(=\mathrm{C}(\mathrm{C} 2=\mathrm{CC}=\mathrm{CC}=\mathrm{C} 2 \mathrm{~S} 1(=\mathrm{O})=\mathrm{O}) \mathrm{O}) \mathrm{C}(=\mathrm{O}) \mathrm{NC} 3=\mathrm{NC}=\mathrm{CS} 3$ \\
\hline 105. & Sulfathiazole & $\begin{array}{l}\text { Topical antibacterial preparation used to treat vaginitis } \\
\text { caused by Haemophilus (Gardnerella) vaginalis bacteria }\end{array}$ & Yes & $\mathrm{NC} 1=\mathrm{CC}=\mathrm{C}(\mathrm{C}=\mathrm{C} 1) \mathrm{S}(=\mathrm{O})(=\mathrm{O}) \mathrm{NC} 1=\mathrm{NC}=\mathrm{CS} 1$ \\
\hline 106. & TAK-580 & Anti-tumor agent & Yes & $\begin{array}{l}\mathrm{C}[\mathrm{C} @ @ \mathrm{H}](\mathrm{NC}(=\mathrm{O}) \mathrm{C} 1=\mathrm{C}(\mathrm{Cl}) \mathrm{C}(\mathrm{N})=\mathrm{NC}=\mathrm{N} 1) \mathrm{C} 1=\mathrm{NC}=\mathrm{C}(\mathrm{S} 1) \mathrm{C}(=\mathrm{O}) \mathrm{NC} 1=\mathrm{NC}=\mathrm{C}(\mathrm{Cl}) \mathrm{C}( \\
=\mathrm{C} 1) \mathrm{C}(\mathrm{F})(\mathrm{F}) \mathrm{F}\end{array}$ \\
\hline 107. & Talarozole & $\begin{array}{l}\text { Investigated for the treatment of psoriasis and cutaneous } \\
\text { inflammation. }\end{array}$ & Yes & $\mathrm{CCC}(\mathrm{CC}) \mathrm{C}(\mathrm{N} 1 \mathrm{C}=\mathrm{NC}=\mathrm{N} 1) \mathrm{C} 1=\mathrm{CC}=\mathrm{C}(\mathrm{NC} 2=\mathrm{NC} 3=\mathrm{CC}=\mathrm{CC}=\mathrm{C} 3 \mathrm{~S} 2) \mathrm{C}=\mathrm{C} 1$ \\
\hline 108. & Tetomilast & $\begin{array}{l}\text { The treatment of Crohn's disease, ulcerative colitis and } \\
\text { Chronic Obstructive Pulmonary Disease (COPD) }\end{array}$ & Yes & $\mathrm{CCOC} 1=\mathrm{C}(\mathrm{OCC}) \mathrm{C}=\mathrm{C}(\mathrm{C}=\mathrm{C} 1) \mathrm{C} 1=\mathrm{NC}(=\mathrm{CS} 1) \mathrm{C} 1=\mathrm{NC}(=\mathrm{CC}=\mathrm{C} 1) \mathrm{C}(\mathrm{O})=\mathrm{O}$ \\
\hline 109. & Thiabendazole & $\begin{array}{l}\text { Active against a variety of nematodes and is the drug of } \\
\text { choice for strongyloidiasis }\end{array}$ & Yes & $\mathrm{N} 1 \mathrm{C} 2=\mathrm{CC}=\mathrm{CC}=\mathrm{C} 2 \mathrm{~N}=\mathrm{C} 1 \mathrm{C} 1=\mathrm{CSC}=\mathrm{N} 1$ \\
\hline 110. & Thiamine & Used to treat low levels of vitamin B1 & Yes & $\mathrm{CC} 1=\mathrm{C}(\mathrm{CCO}) \mathrm{SC}=[\mathrm{N}+] 1 \mathrm{CC} 1=\mathrm{CN}=\mathrm{C}(\mathrm{C}) \mathrm{N}=\mathrm{C} 1 \mathrm{~N}$ \\
\hline 111. & Thiohexam & Allergen and dermatological sensitizer & Yes & $\mathrm{C} 1 \mathrm{CCC}(\mathrm{CC} 1) \mathrm{NSC} 1=\mathrm{NC} 2=\mathrm{CC}=\mathrm{CC}=\mathrm{C} 2 \mathrm{~S} 1$ \\
\hline 112. & Tioxidazole & Antiparasitic activity & Yes & $\mathrm{CCCOC} 1=\mathrm{CC}=\mathrm{C} 2 \mathrm{~N}=\mathrm{C}(\mathrm{NC}(=\mathrm{O}) \mathrm{OC}) \mathrm{SC} 2=\mathrm{C} 1$ \\
\hline 113. & Tozadenant & $\begin{array}{l}\text { Has been under trials for the treatment of Parkinson's disease } \\
\text { as an adjunctive therapy with levodopa }\end{array}$ & Yes & $\mathrm{COC} 1=\mathrm{C} 2 \mathrm{~N}=\mathrm{C}(\mathrm{NC}(=\mathrm{O}) \mathrm{N} 3 \mathrm{CCC}(\mathrm{C})(\mathrm{O}) \mathrm{CC} 3) \mathrm{SC} 2=\mathrm{C}(\mathrm{C}=\mathrm{C} 1) \mathrm{N} 1 \mathrm{CCOCC} 1$ \\
\hline 114. & Troriluzole & Under investigation for spinocerebellar ataxia & Yes & $\mathrm{CN}(\mathrm{CC}(=\mathrm{O}) \mathrm{NC} 1=\mathrm{NC} 2=\mathrm{C}(\mathrm{S} 1) \mathrm{C}=\mathrm{C}(\mathrm{OC}(\mathrm{F})(\mathrm{F}) \mathrm{F}) \mathrm{C}=\mathrm{C} 2) \mathrm{C}(=\mathrm{O}) \mathrm{CNC}(=\mathrm{O}) \mathrm{CN}$ \\
\hline 115. & VM4-037 & PET Agent for imaging of renal cell carcinoma & No & $\begin{array}{l}\mathrm{CC}(\mathrm{C})[\mathrm{C} @ \mathrm{H}](\mathrm{N} 1 \mathrm{C}=\mathrm{C}(\mathrm{COC} 2=\mathrm{CC} 3=\mathrm{C}(\mathrm{C}=\mathrm{C} 2) \mathrm{N}=\mathrm{C}(\mathrm{S} 3) \mathrm{S}(\mathrm{N})(=\mathrm{O})=\mathrm{O}) \mathrm{N}=\mathrm{N} 1) \mathrm{C}(=\mathrm{O}) \mathrm{N}[ \\
\mathrm{C} @ @ \mathrm{H}](\mathrm{CC} 1=\mathrm{CC}=\mathrm{C}(\mathrm{OCCF}) \mathrm{C}=\mathrm{C} 1) \mathrm{C}(\mathrm{O})=\mathrm{O}\end{array}$ \\
\hline 116. & Varlitinib & $\begin{array}{l}\text { Possess significant anti-tumor activity in preclinical models } \\
\text { of human breast, lung, and epidermal carcinoma tumors }\end{array}$ & Yes & $\begin{array}{l}\mathrm{C}[\mathrm{C} @ @ \mathrm{H}] 1 \mathrm{COC}(\mathrm{NC} 2=\mathrm{CC}=\mathrm{C} 3 \mathrm{~N}=\mathrm{CN}=\mathrm{C}(\mathrm{NC} 4=\mathrm{CC}=\mathrm{C}(\mathrm{OCC} 5=\mathrm{NC}=\mathrm{CS} 5) \mathrm{C}(\mathrm{Cl})=\mathrm{C} 4) \mathrm{C} \\
3=\mathrm{C} 2)=\mathrm{N} 1\end{array}$ \\
\hline 117. & Vedroprevir & $\begin{array}{l}\text { As been investigated for the treatment of Hepatitis C, } \\
\text { Chronic. }\end{array}$ & No & $\begin{array}{l}\mathrm{CC}[\mathrm{C} @ @ \mathrm{H}] 1 \mathrm{C}[\mathrm{C} @] 1(\mathrm{NC}=\mathrm{O})[\mathrm{C} @ @ \mathrm{H}] 1 \mathrm{C}[\mathrm{C} @ \mathrm{H}](\mathrm{CN} 1 \mathrm{C}(=\mathrm{O})[\mathrm{C} @ @ \mathrm{H}](\mathrm{NC}=\mathrm{O}) \mathrm{O}[\mathrm{C} \\
@ @ \mathrm{H}] 1 \mathrm{C}[\mathrm{C} @ \mathrm{H}] 2 \mathrm{C}[\mathrm{C} @ @ \mathrm{H}] 2 \mathrm{C} 1) \mathrm{C}(\mathrm{C})(\mathrm{C}) \mathrm{C}) \mathrm{OC} 1=\mathrm{C} 2 \mathrm{C}=\mathrm{CC}(\mathrm{OCCN} 3 \mathrm{CCOCC} 3)=\mathrm{C}( \\
\mathrm{Cl}) \mathrm{C} 2=\mathrm{NC}(=\mathrm{C} 1) \mathrm{C} 1=\mathrm{CSC}(\mathrm{NC}(\mathrm{C}) \mathrm{C})=\mathrm{N} 1) \mathrm{C}(\mathrm{O})=\mathrm{O}\end{array}$ \\
\hline 118. & Venglustat & $\begin{array}{l}\text { The treatment of Parkinson's Disease, Gaucher Disease, and } \\
\text { Fabry Disease. }\end{array}$ & Yes & $\mathrm{CC}(\mathrm{C})(\mathrm{NC}(=\mathrm{O}) \mathrm{O}[\mathrm{C} @ @ \mathrm{H}] 1 \mathrm{CN} 2 \mathrm{CCC} 1 \mathrm{CC} 2) \mathrm{C} 1=\mathrm{CSC}(=\mathrm{N} 1) \mathrm{C} 1=\mathrm{CC}=\mathrm{C}(\mathrm{F}) \mathrm{C}=\mathrm{C} 1$ \\
\hline 119. & Vosaroxin & Under investigation for the treatment of leukemia, Myeloid & Yes & $\begin{array}{l}\mathrm{CN}[\mathrm{C} @ \mathrm{H}] 1 \mathrm{CN}(\mathrm{C}[\mathrm{C} @ @ @ \mathrm{H}] 1 \mathrm{OC}) \mathrm{C} 1=\mathrm{CC}=\mathrm{C} 2 \mathrm{C}(=\mathrm{O}) \mathrm{C}(=\mathrm{CN}(\mathrm{C} 3=\mathrm{NC}=\mathrm{CS} 3) \mathrm{C} 2=\mathrm{N} 1) \mathrm{C}(\mathrm{O} \\
)=\mathrm{O}\end{array}$ \\
\hline 120. & Ziritaxestat & Under investigation for systemic sclerosis & No & $\begin{array}{l}\mathrm{CCC} 1=\mathrm{C}(\mathrm{N}(\mathrm{C}) \mathrm{C} 2=\mathrm{NC}(=\mathrm{C}(\mathrm{S} 2) \mathrm{C} \# \mathrm{~N}) \mathrm{C} 2=\mathrm{CC}=\mathrm{C}(\mathrm{F}) \mathrm{C}=\mathrm{C} 2) \mathrm{N} 2 \mathrm{C}=\mathrm{C}(\mathrm{C}=\mathrm{C}(\mathrm{C}) \mathrm{C} 2=\mathrm{N} 1) \mathrm{N} 1 \mathrm{C} \\
\mathrm{CN}(\mathrm{CC}(=\mathrm{O}) \mathrm{N} 2 \mathrm{CC}(\mathrm{O}) \mathrm{C} 2) \mathrm{CC} 1\end{array}$ \\
\hline
\end{tabular}

${ }^{\circledR}$ The authors thank DrugBank for making its data bank publicly available at https://go.drugbank.com/. 
Table S2. List of nucleophilic residues present in the vicinity of active site in CYP 2C9, 2D6, 3A4, 2C19 and 2E1 (up to $6 \AA$ ).

\begin{tabular}{|c|c|c|c|c|c|}
\hline Residues & $\begin{array}{c}\text { CYP2C9 } \\
(1 \mathrm{R9O})\end{array}$ & $\begin{array}{c}\text { CYP2D6 } \\
\text { (3TBG) }\end{array}$ & $\begin{array}{c}\text { CYP3A4 } \\
\text { (5VCC) }\end{array}$ & $\begin{array}{l}\text { CYP2C19 } \\
\text { (4GQS) }\end{array}$ & $\begin{array}{c}\text { CYP2E1 } \\
(3 T 3 Z)\end{array}$ \\
\hline Up to $4 \AA$ & S135, S304 & S304, T309 & $\begin{array}{l}\text { T307, T309, } \\
\text { T310, } \\
\text { S119, S311, } \\
\text { S312 }\end{array}$ & $\begin{array}{l}\text { S209, S478 } \\
\text { T301, T304 } \\
\text { N107, }\end{array}$ & $\begin{array}{l}\text { T301, T303, S366, } \\
\text { N367 }\end{array}$ \\
\hline$<5 \AA$ & $\begin{array}{l}\text { T301, R108, } \\
\text { S289 }\end{array}$ & C443 & Q484, M371 & $\begin{array}{l}\text { S303, T302, } \\
\text { T304, T305 } \\
\text { N204, N474 } \\
\text { R206,R307 }\end{array}$ & $\begin{array}{l}\text { Q216, N219, N220, } \\
\text { T304, T306 }\end{array}$ \\
\hline$<6 \AA$ & $\begin{array}{l}\text { S116, S209, } \\
\text { S365, } \\
\text { R97, R101, } \\
\text { R115, R132, } \\
\text { R296, R441, } \\
\text { K200, T299 }\end{array}$ & $\begin{array}{l}\text { K214, R221, } \\
\text { T310, T375, } \\
\text { S437, M307 }\end{array}$ & $\begin{array}{l}\text { T224, R105, } \\
\text { R106 R212, } \\
\text { R372, R375 }\end{array}$ & $\begin{array}{l}\text { S115, S365, } \\
\text { T210, T290, } \\
\text { T320, T299 } \\
\text { N202, N204 }\end{array}$ & $\begin{array}{l}\text { R100, N206, S211, } \\
\text { T392, H370 }\end{array}$ \\
\hline
\end{tabular}


Table S3. The relative free energies $(\Delta G)$ and the global electrophilicity indices $(\omega)$ of the reactive metabolites. The activation energies $\left(E_{\mathrm{a}}\right)$ for the reaction of the $\mathbf{R M}$ with $\mathrm{MeO}^{-}$.

\begin{tabular}{|c|c|c|c|}
\hline & & ATZ & \\
\hline $\mathrm{RM}$ & $\begin{array}{c}\text { Relative } \\
\text { Energy } \\
(\Delta G)^{\mathrm{a}}\end{array}$ & $\begin{array}{l}\text { Electrophilicity } \\
(\omega)\end{array}$ & $E_{\mathrm{a}}\left(\mathrm{MeO}^{-}\right)^{\mathrm{a}}$ \\
\hline TZ/ATZ & & 0.99 & \\
\hline M1 & 0.00 & 1.28 & 7.21 \\
\hline M2 & 21.43 & 2.13 & 4.22 \\
\hline M3 & 27.74 & 1.48 & 4.34 \\
\hline M4 & 42.12 & 1.95 & 9.81 \\
\hline I5 & -18.65 & 1.27 & 19.84 \\
\hline I6 & -39.77 & 1.49 & 8.54 \\
\hline 17 & -14.49 & 0.98 & 19.76 \\
\hline I8 & -32.88 & 1.46 & 8.21 \\
\hline 19 & -17.00 & 2.66 & 6.12 \\
\hline I10 & -11.84 & 3.90 & 3.12 \\
\hline I11 & -15.77 & 4.19 & 3.01 \\
\hline I12 & -- & -- & -- \\
\hline I13 & -- & -- & -- \\
\hline I14 & -- & -- & -- \\
\hline D1 & 17.63 & 4.67 & \\
\hline D2 & 19.92 & 1.93 & \\
\hline D3 & 0.00 & 2.01 & \\
\hline D4 & 32.10 & 2.08 & \\
\hline D5 & 15.81 & 2.39 & \\
\hline D6 & 27.48 & 3.78 & \\
\hline D7 & 67.76 & 2.76 & \\
\hline D8 & 78.44 & 2.76 & \\
\hline D9 & -- & -- & -- \\
\hline D10 & -- & -- & -- \\
\hline
\end{tabular}


Table S4. The absolute energies (Gibbs free energy) in Hartree of the various species employed in the manuscript.The values were obtained using B3LYP(SCRF)/6-311++G(d,p)// B3LYP/6-31+G(d) basis set $(\varepsilon=5.9)$.

\begin{tabular}{|c|c|}
\hline Structure name & Gibbs free energy in Hartree \\
\hline RC & -2154.589381 \\
\hline TS-E & -2154.567654 \\
\hline PC-E & -2154.629892 \\
\hline TS-N & -2154.557197 \\
\hline PC-N & -2154.615346 \\
\hline TS-S & -2154.560849 \\
\hline PC-S & -2154.596006 \\
\hline TS-A & -2154.566172 \\
\hline PC-A & -2154.578779 \\
\hline Fe-porphyrin & -1510.425167 \\
\hline${ }^{4} \mathbf{R C}$ & -2154.595162 \\
\hline $\begin{array}{l}{ }^{4} \mathrm{TS}-\mathrm{E} \\
\end{array}$ & -2154.567292 \\
\hline${ }^{4} \mathrm{PC}-\mathrm{E}$ & -2154.626192 \\
\hline${ }^{4} \mathrm{TS}-\mathrm{N}$ & -2154.558287 \\
\hline${ }^{4} \mathrm{PC}-\mathrm{N}$ & -2154.615651 \\
\hline${ }^{4} \mathrm{TS}-\mathrm{S}$ & -2154.559487 \\
\hline${ }^{4}$ PC-S & -2154.600599 \\
\hline RC(ATZ) & -2209.93438 \\
\hline TS-E(ATZ) & -2209.92297 \\
\hline TS-S(ATZ) & -2209.92167 \\
\hline TS-N(ATZ) & -2209.91505 \\
\hline PC-E(ATZ) & -2209.99408 \\
\hline PC-S(ATZ) & -2209.96655 \\
\hline PC-N(ATZ) & -2209.97346 \\
\hline RC-N(ATZ) & -2209.9327384 \\
\hline TS-N-hydroxylation(ATZ) & -2209.9207179 \\
\hline TS-N-radical(ATZ) & -2209.9385889 \\
\hline RC(AMTZ) & -2249.23885 \\
\hline TS-E(AMTZ) & -2249.212348 \\
\hline PC-E(AMTZ) & -2249.286911 \\
\hline TS-H(AMTZ) & -2249.21498 \\
\hline Int-1(AMTZ) & -2249.241288 \\
\hline PC-H(AMTZ) & -2249.311364 \\
\hline TS-S(AMTZ) & -2249.211427 \\
\hline PC-S(AMTZ) & -2249.259608 \\
\hline TS-N(AMTZ) & -2249.20791 \\
\hline PC-N(AMTZ) & -2249.264359 \\
\hline M1 & -644.202472 \\
\hline M2 & -644.163817 \\
\hline $\mathbf{M 3}$ & -644.180564 \\
\hline
\end{tabular}




\begin{tabular}{|c|c|}
\hline M4 & -644.156507 \\
\hline I5 & -644.250192 \\
\hline I6 & -644.258904 \\
\hline I7 & -644.235529 \\
\hline I8 & -644.256771 \\
\hline I9 & -644.254351 \\
\hline I10 & -644.214328 \\
\hline I11 & -644.218264 \\
\hline I12 & -644.25161 \\
\hline I13 & -644.270119 \\
\hline I14 & -644.259984 \\
\hline DI1 & -719.332905 \\
\hline DI2 & -719.39051 \\
\hline DI3 & -719.416228 \\
\hline DI4 & -719.378598 \\
\hline DI5 & -719.401073 \\
\hline DI6 & -719.404767 \\
\hline DI7 & -719.325737 \\
\hline DI8 & -719.312034 \\
\hline DI9 & -719.416036 \\
\hline DI10 & -719.420415 \\
\hline ATZ & -624.375519 \\
\hline ATZ-M1 & -699.560085 \\
\hline ATZ-M2 & -699.52593 \\
\hline ATZ-M3 & -699.515878 \\
\hline ATZ-M4 & -699.492965 \\
\hline ATZ-I5 & -699.589799 \\
\hline ATZ-I6 & -699.623455 \\
\hline ATZ-I7 & -699.583174 \\
\hline ATZ-I8 & -699.612476 \\
\hline ATZ-I9 & -699.587183 \\
\hline ATZ-I10 & -699.578953 \\
\hline ATZ-I11 & -699.585212 \\
\hline ATZ_D1 & -774.759263 \\
\hline ATZ_D2 & -774.755609 \\
\hline ATZ_D3 & -774.787355 \\
\hline ATZ_D4 & -774.736208 \\
\hline ATZ_D5 & -774.762154 \\
\hline ATZ_D6 & -774.743564 \\
\hline ATZ_D7 & -774.679379 \\
\hline ATZ_D8 & -774.662353 \\
\hline TS1 & -644.1792974 \\
\hline $\mathrm{MeO}^{-}$ & -115.098638 \\
\hline TS2 & -759.2859011 \\
\hline M1-OH & -759.347661 \\
\hline TS3 & -1082.2942759 \\
\hline M1-SH & -1082.364645 \\
\hline TS4 & -720.576268 \\
\hline
\end{tabular}




\begin{tabular}{|l|l|}
\hline M1-D & -720.642096 \\
\hline TS5 & -720.572876 \\
\hline TS5 & -797.02113 \\
\hline HM1 & -720.624822 \\
\hline TS6 & -720.6418834 \\
\hline TS6 & -797.0376817 \\
\hline TS7 & -720.581773 \\
\hline TS7 & -797.021675 \\
\hline HM2 & -720.642409 \\
\hline TS8 & -720.628490 \\
\hline TS8 & -797.0547717 \\
\hline M5 & -492.836324 \\
\hline M6 & -227.814533 \\
\hline
\end{tabular}




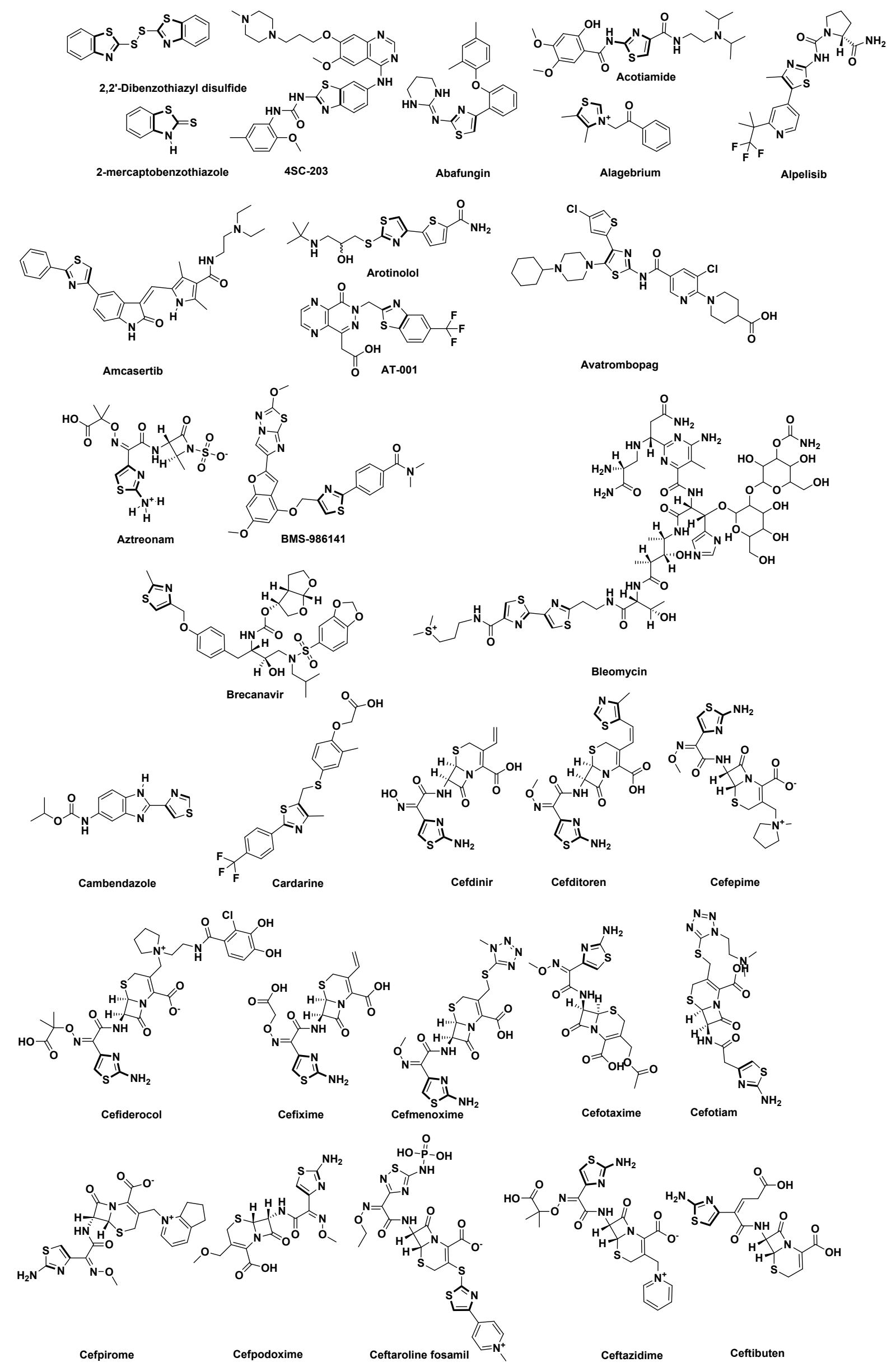



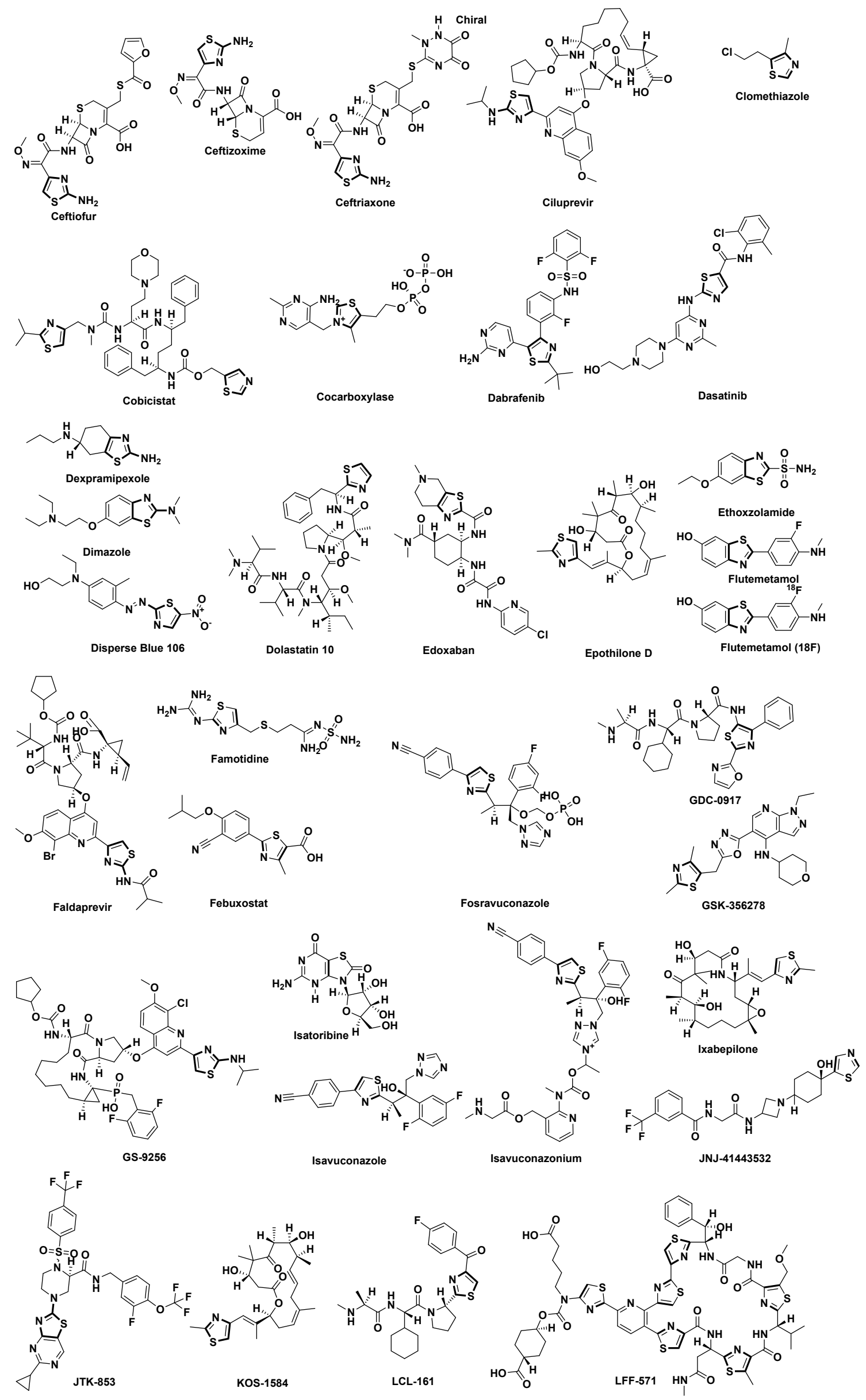
(n)

LY-2608204

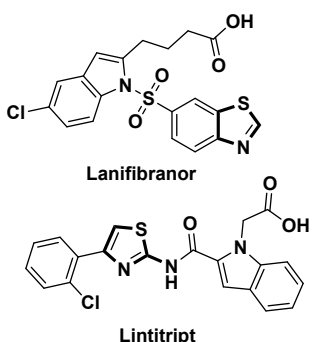

Lintitript

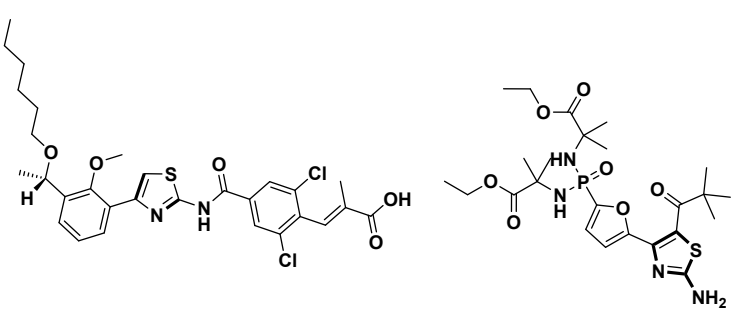

Lusutrombopag

MB-07803

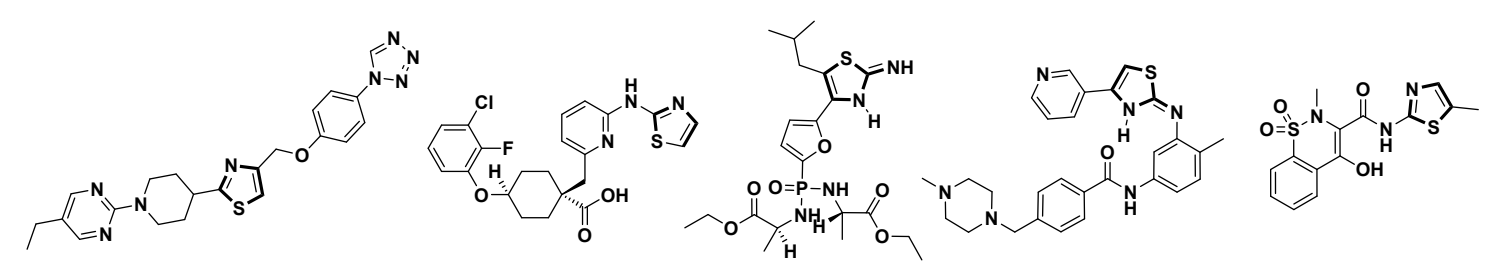

MBX-2982

MK-5108

Managlinat dialanetil

Masitinib

Meloxicam
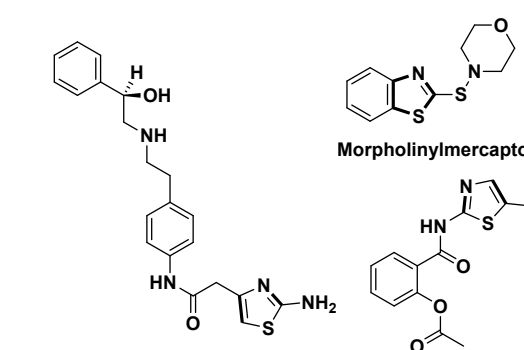

MorpholinyImercaptobenzothiazole
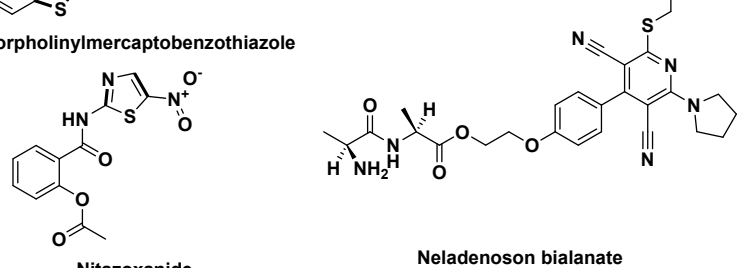

Mirabegron

Nitazoxanide

Neladenoson bialanate
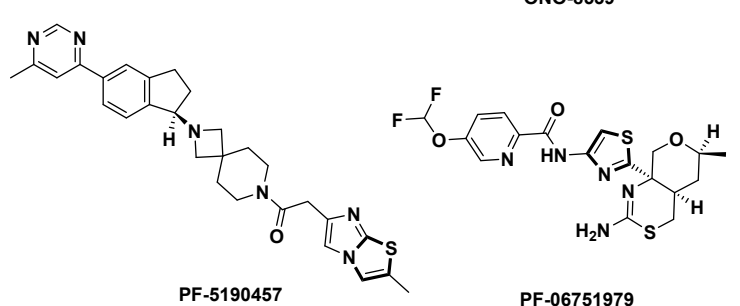

Oprozomib

PF-05089771

PF-5190457

PF-06751979

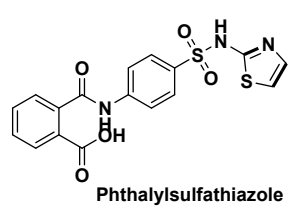

${ }_{\mathrm{N}}^{\mathrm{HO}}=\mathrm{NH}$<smiles>CCCNC1CCc2nc(N)sc2C1</smiles>

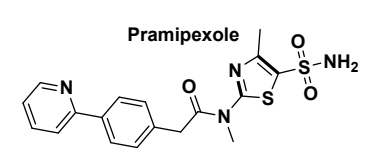

Pittsburgh Compound B

Pritelivir
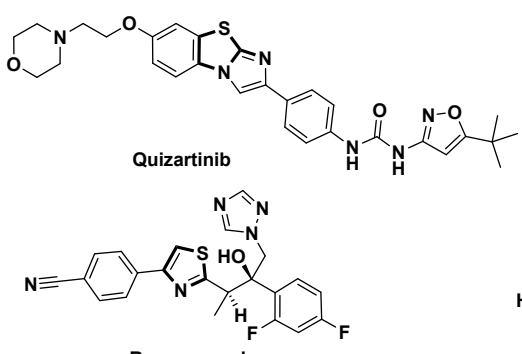

Ravuconazole
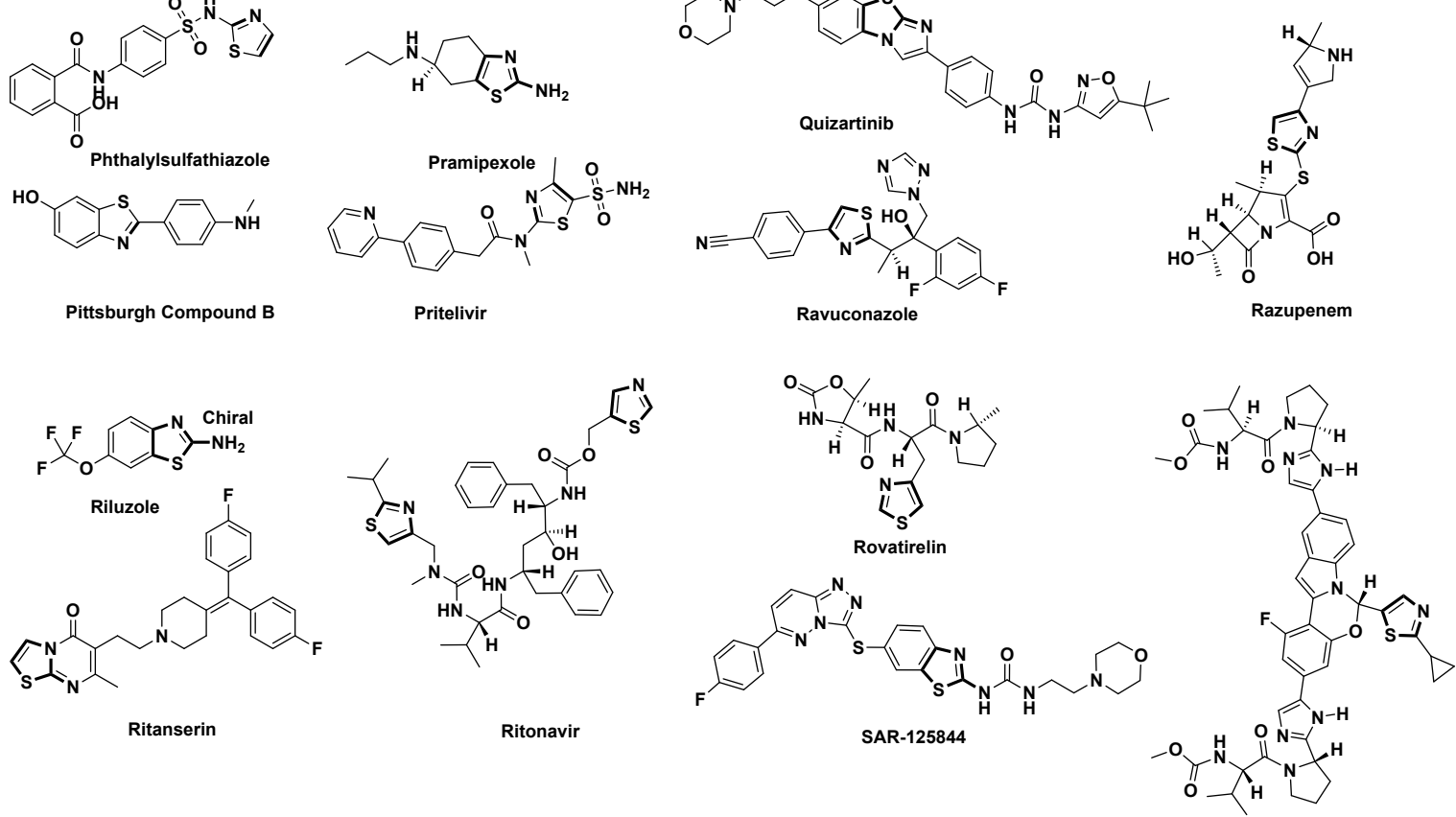

Ruzasvir 


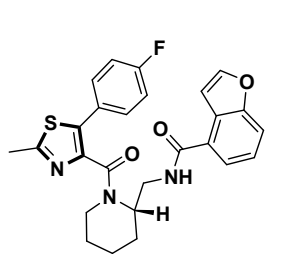

SB-649868

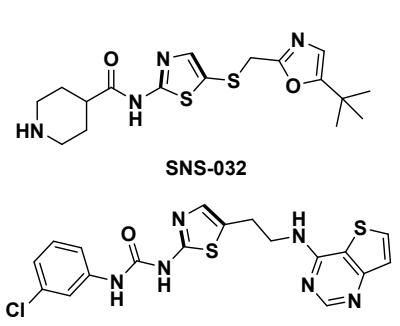

SNS-314

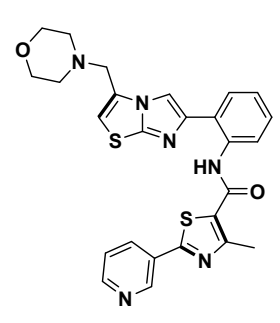

SRT-2104

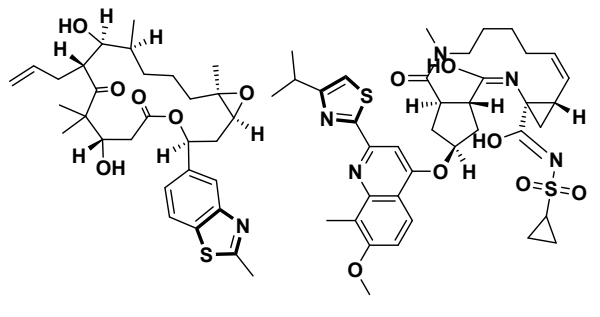

Sagopilone

Simeprevir<smiles>CNc1ccc(-c2nc3ccc(F)cc3s2)cc1</smiles>

Snubh-nm-333 F-18

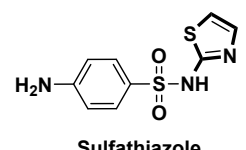

Sulfathiazole
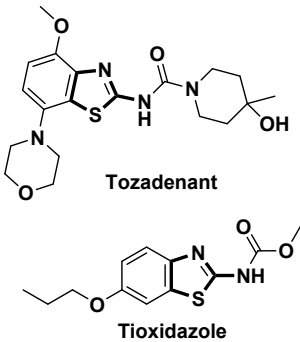

${ }^{\mathrm{H}^{2}} \underbrace{\mathrm{O}^{2}}$<smiles>CCNc1nc2ccc(OC(F)(F)F)cc2s1</smiles>

Troriluzole
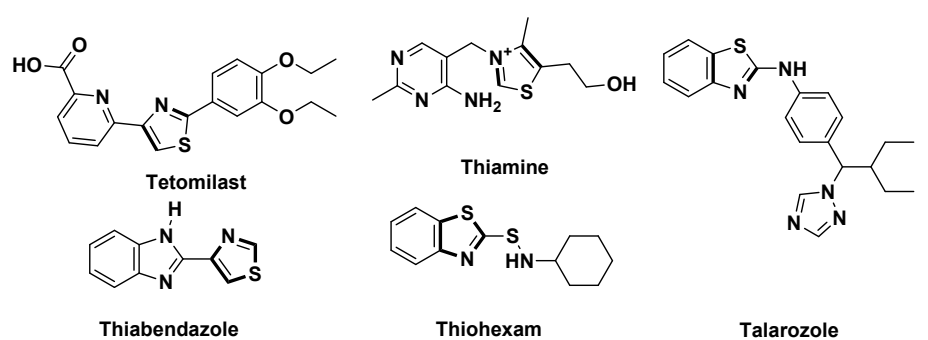

Talarozole

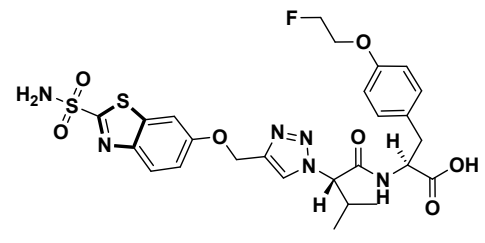

VM4-037
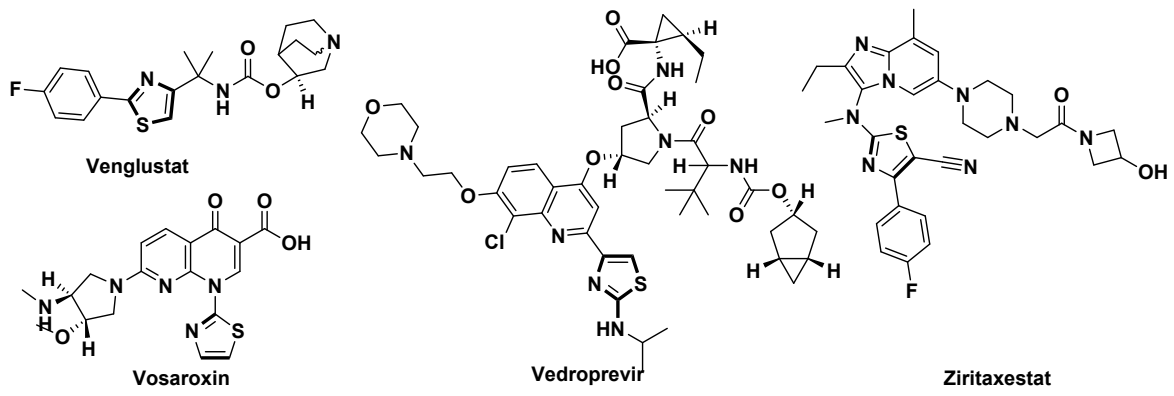

Figure S1. 2D structures of thiazole compounds (as listed in TableS1). 


\section{Epoxidation}
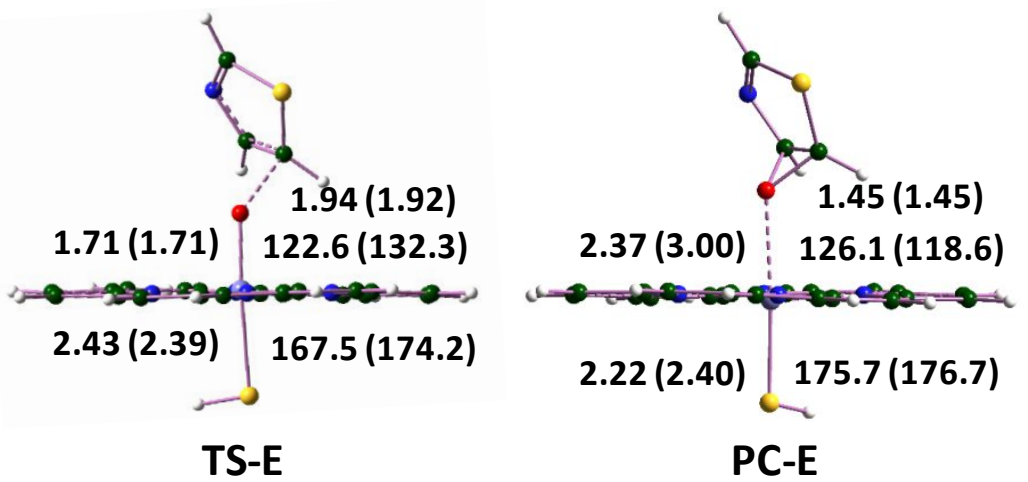

S-Oxidation
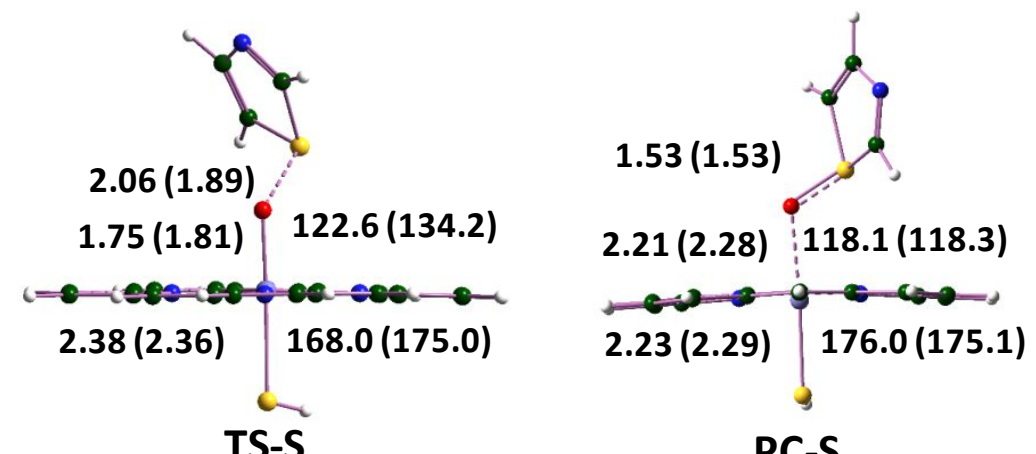

PC-S

\section{N-Oxidation}

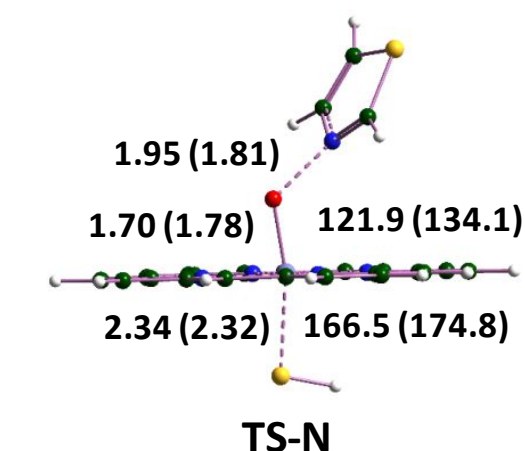

Oxaziridine Formation

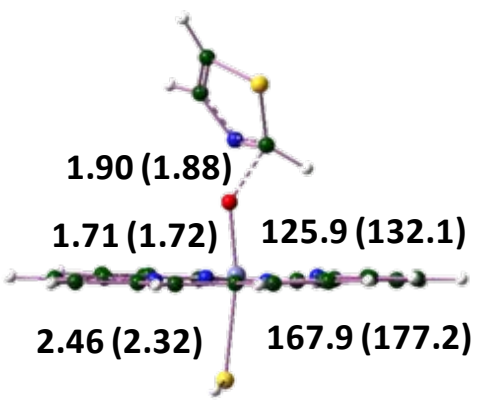

TS-A
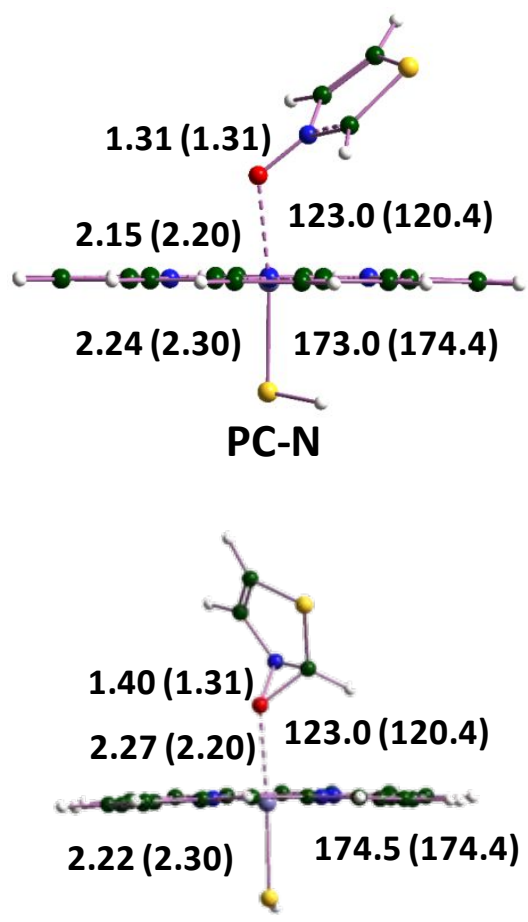

PC-A

Figure S2. Optimized geometries of transition states (TS-E, TS-S, TS-N, TS-A) and product complexes (PCE, PC-S, PC-N, PC-A) along the various reaction pathways of thiazole. The values are on the doublet (quartet) potential energy surface. All bond lengths are in Ångstrom $(\AA)$ and bond angles are in degrees $\left(^{\circ}\right)$. 


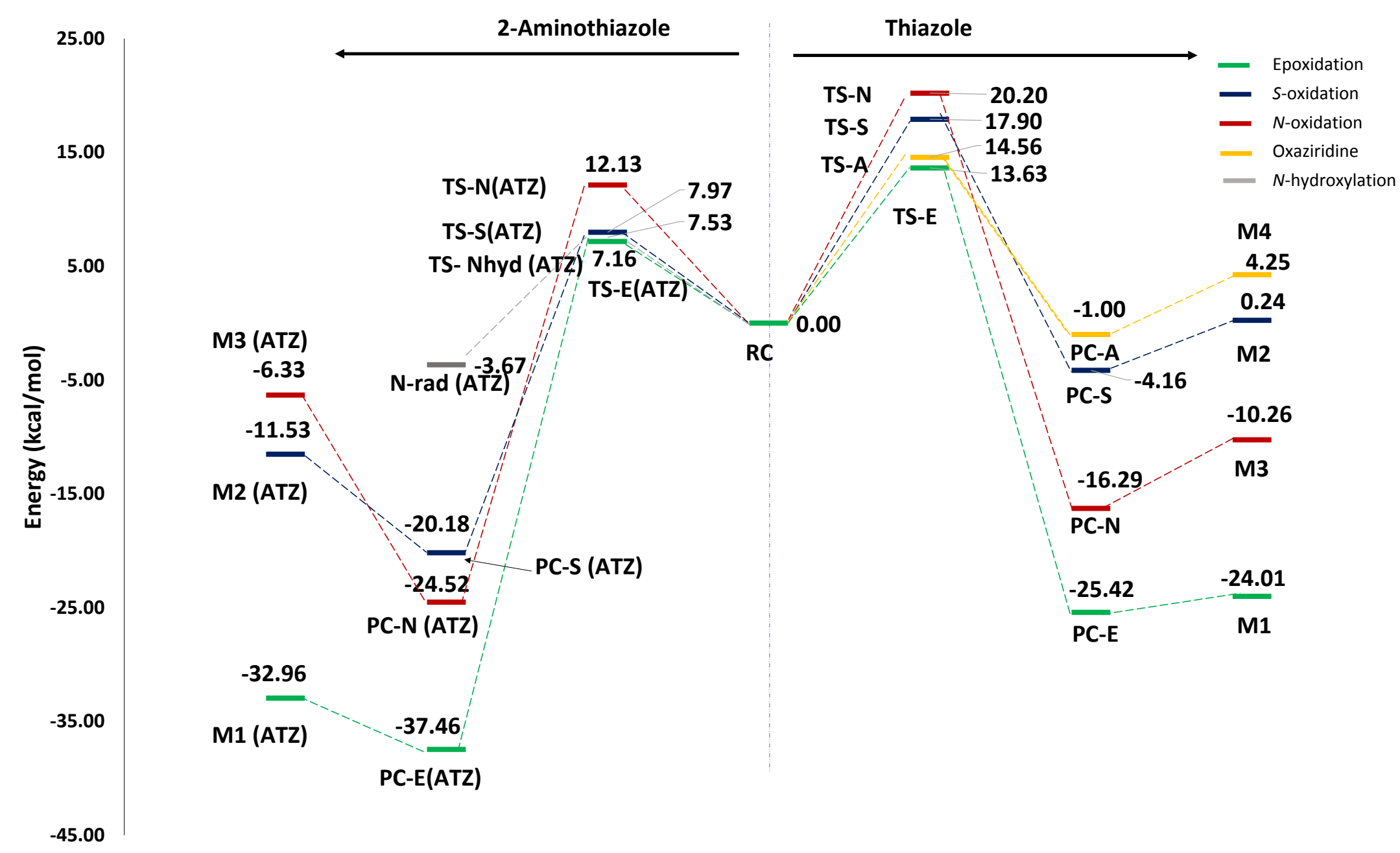

Figure S3. Energy profile comparison between TZ (right side) and ATZ (left side) (epoxidation, $S$-oxidation, $N$-oxidation, oxaziridine and $N$-hydroxylation formation in the presence of $\mathrm{Cpd}$ I) on the doublet potential energy surface of Cpd I. All the values are obtained using B3LYP(SCRF)/6$311++\mathrm{G}(\mathrm{d}, \mathrm{p}) / / \mathrm{B} 3 \mathrm{LYP} / 6-31+\mathrm{G}(\mathrm{d})$. 


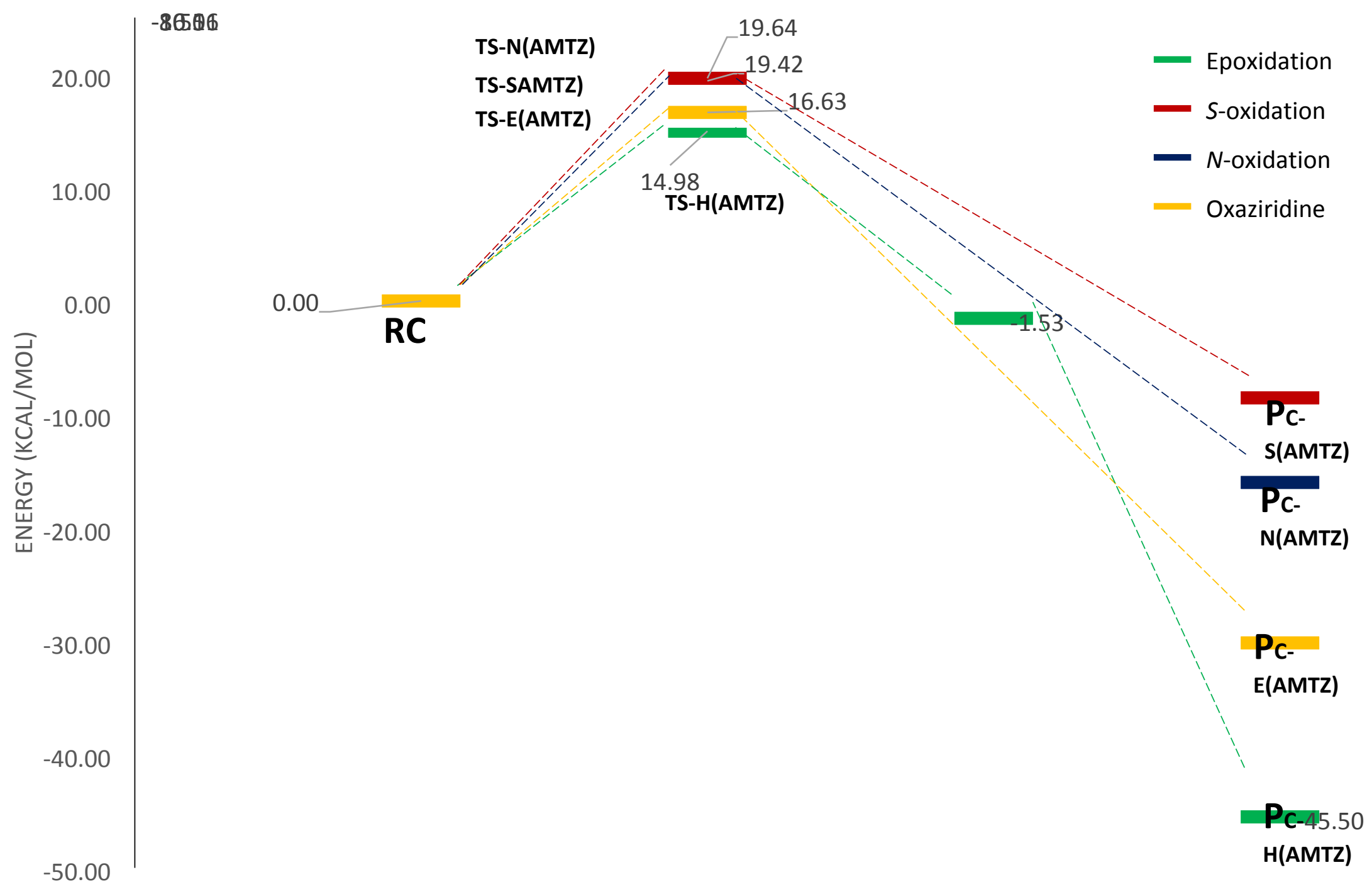

Figure S4. Potential energy profile of AMTZ (hydroxylation, epoxidation, $S$-oxidation, and $N$-oxidation) on the doublet state of Cpd I. All the values are obtained using B3LYP(SCRF)/6-311++G(d,p)//B3LYP/6-31+G(d) level of quantum chemistry. 


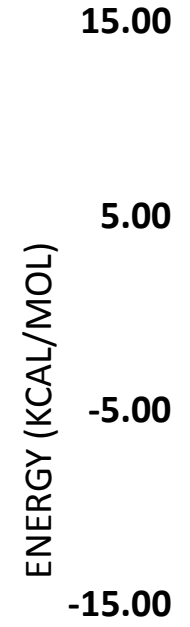

$-25.00$

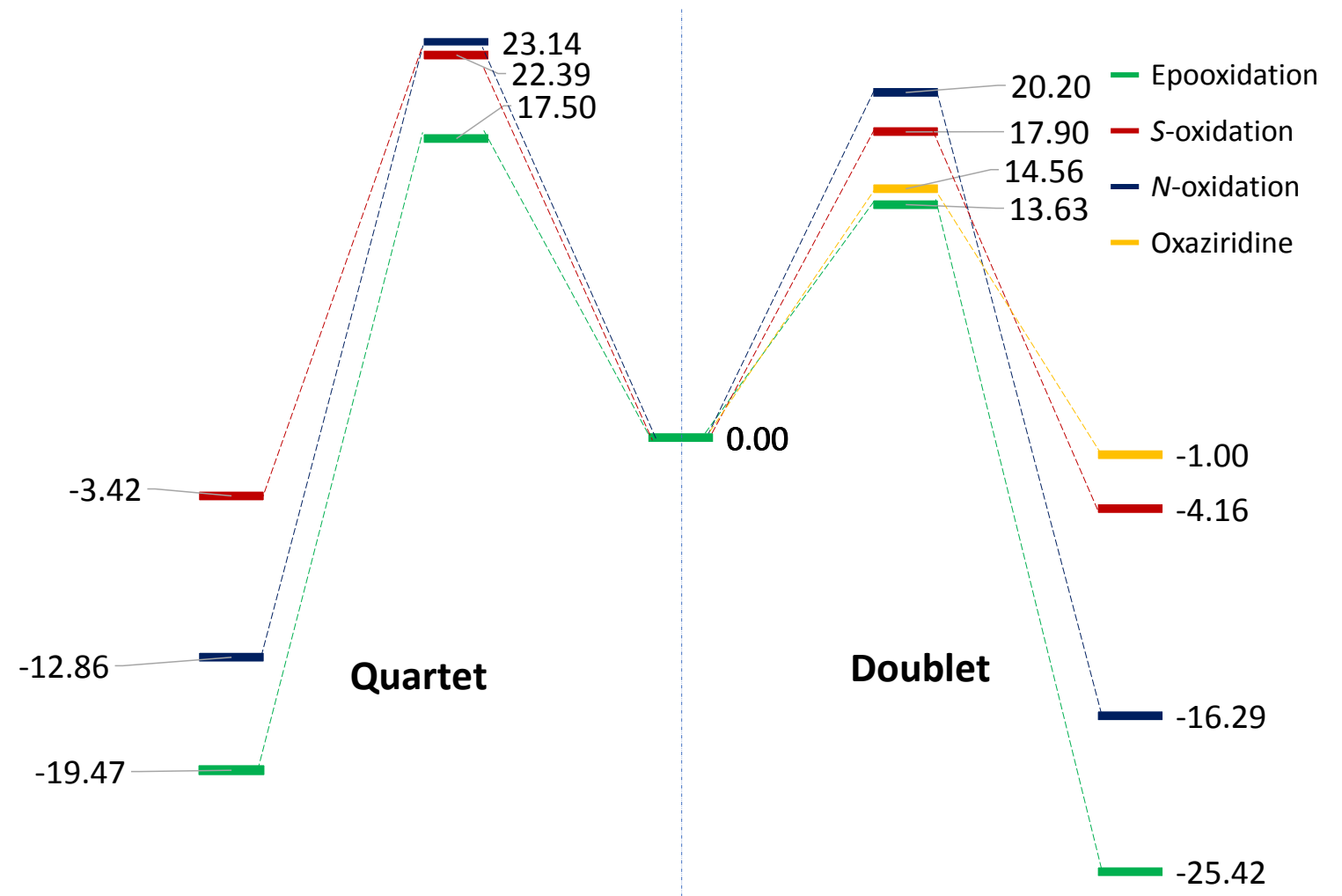

$-35.00$

Figure S5. A comparative potential energy profile of TZ (epoxidation, $S$-oxidation, and $N$-oxidation) on the doublet (right) quartet state (left) of Cpd I. All the values are obtained using B3LYP(SCRF)/6-311++G(d,p)//B3LYP/6-31+G(d) level of quantum chemistry. Since the values along the quartet PE surface are consistently higher than that of the double PE surface, the discussion in the main text is limited to the results according to doublet PE surface. 

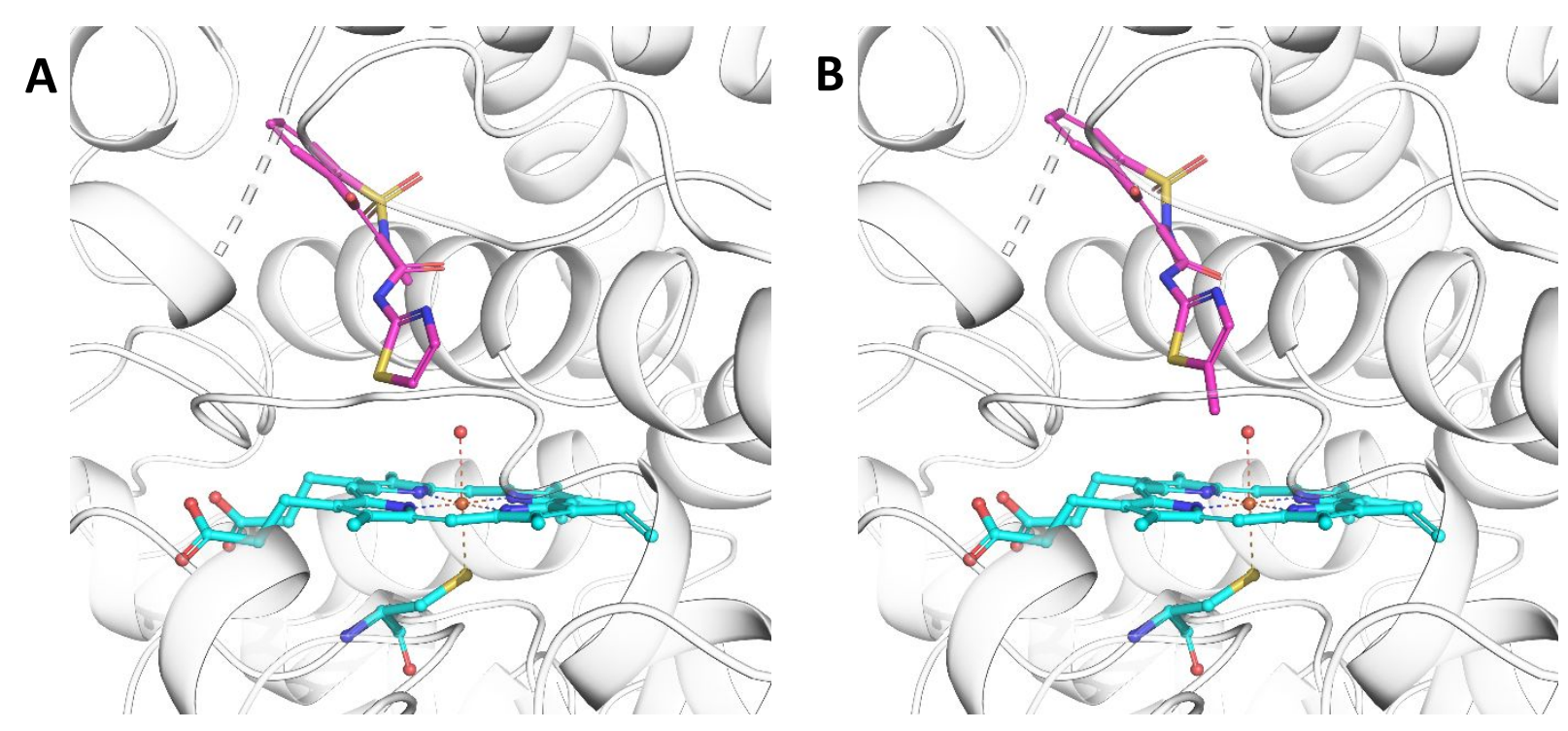

Figure S6. The docking poses of sudoxicam (A) favoring epoxidation, $\mathrm{C}^{5}$ atom of the thiazole ring is pointed toward the $\mathrm{Fe}=\mathrm{O}$ center and meloxicam $(\mathbf{B})$ favoring hydroxylation, the methyl group at $\mathrm{C}^{5}$ of thiazole ring is pointed towards the $\mathrm{Fe}=\mathrm{O}$ center in active site of CYP3A4. 


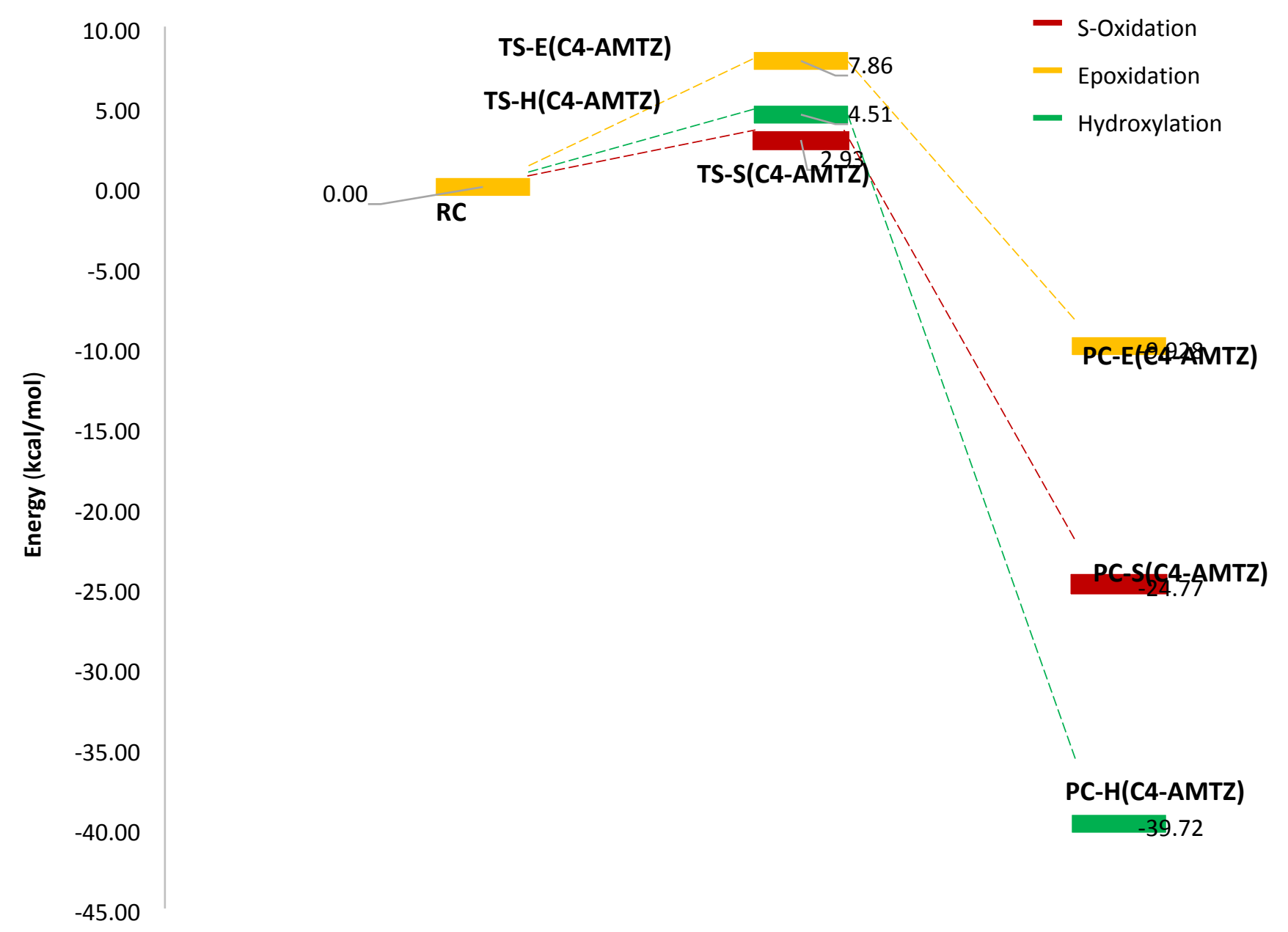

Figure S7. Potential energy profile diagram of C4-AMTZ (hydroxylation, epoxidation and S-oxidation) on the doublet state of Cpd I. All the values are obtained using B3LYP(SCRF)/6-311++G(d,p)//B3LYP/6-31+G(d) level of quantum chemistry. 


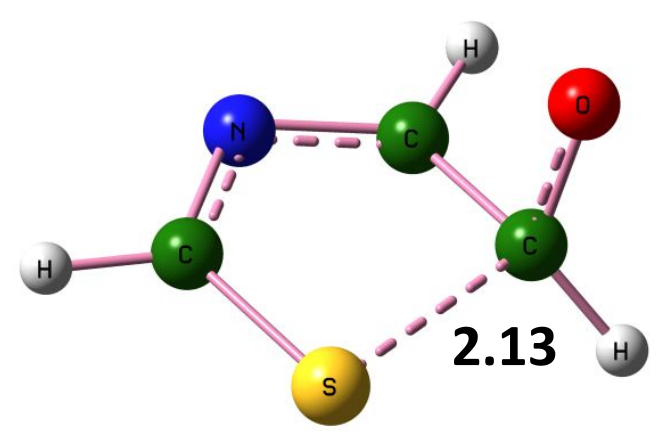

TS1

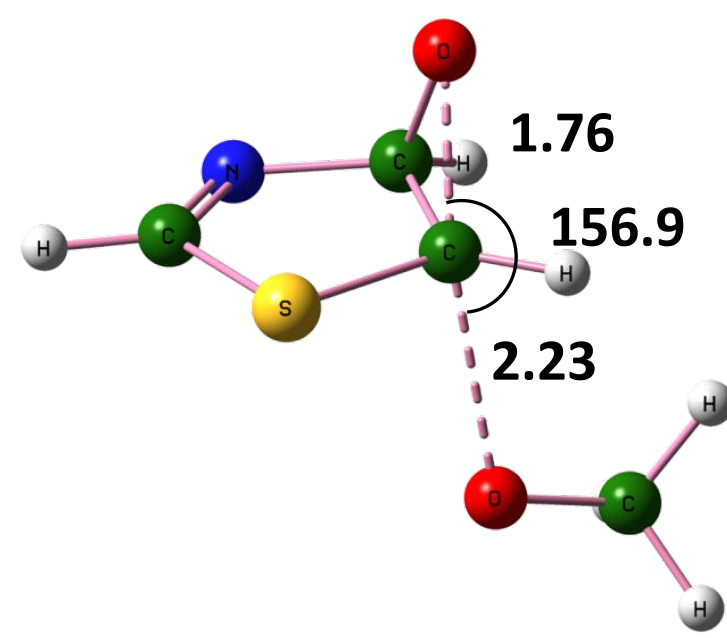

TS2

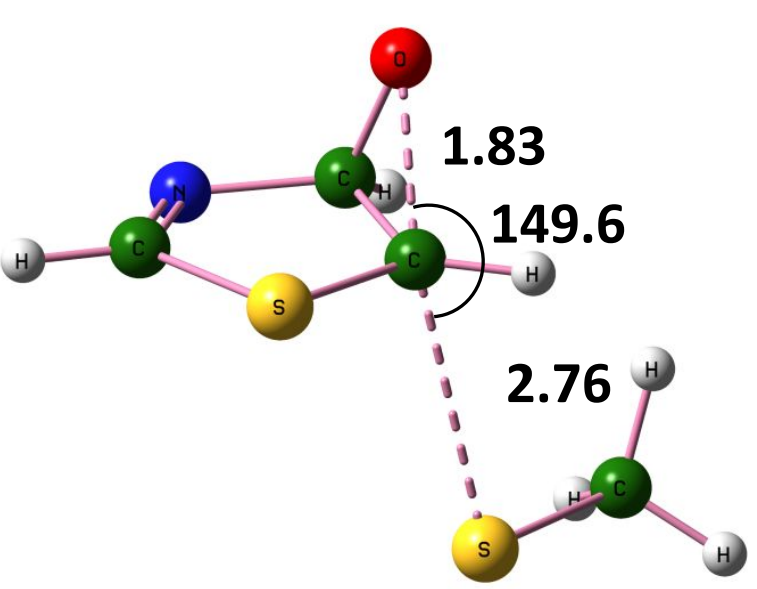

TS3

Figure S8. The transition states involved in the rearrangement reaction from $\mathbf{M 1}$ to I10and nucleophilic reaction between $\mathbf{M 1}^{\text {and }} \mathrm{MeO}^{-}$(TS2) and MeS ${ }^{-}$ (TS3). All of the distances are in angstroms $(\AA)$ and angles are in degree $\left(^{\circ}\right)$. 
A

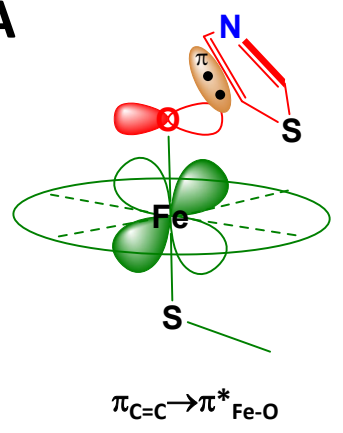

B

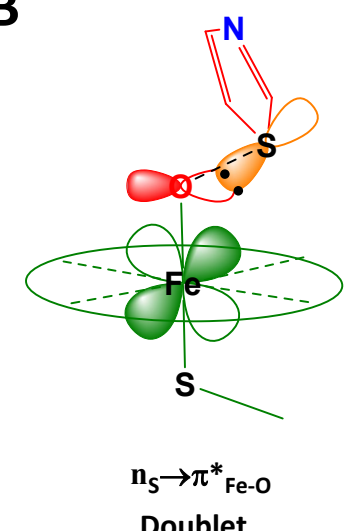

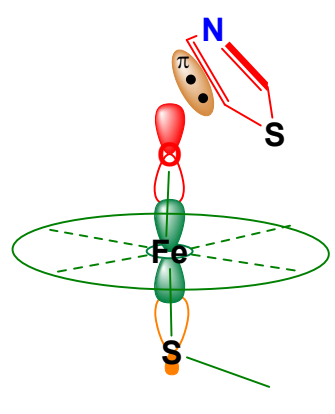

$\pi_{\mathrm{C}=\mathrm{C}} \rightarrow \sigma^{*}{ }_{\mathrm{Fe}-\mathrm{O}}$

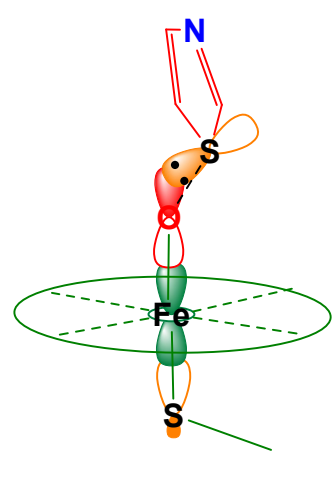

$\mathbf{n}_{\mathrm{S}} \rightarrow \sigma^{*}{ }_{\mathrm{Fe}-\mathrm{O}}$

Quartet

Figure S9. Participation of orbitals in the electron transfer during epoxidation and $S$-oxidation pathways. (a) Cartoon representation of orbital overlap of the $\pi$-bond with the $\pi *_{x z}$ orbital of heme in the doublet state (left) and with $\sigma *_{z^{2}}$ of heme in the quartet state (right). (b) Cartoon representation of the orbital overlap of $S$-lone pair with $\pi *_{\mathrm{xz}}$ of heme in the doublet state (left) and with $\sigma *_{\mathrm{z}^{2}}$ of heme in the quartet state (right). 
